\title{
Radial Injector Mixing Effects on Detonation Zone Position in Rotating Detonation Engine
}

\author{
Malik R. Washington \\ A thesis \\ submitted in partial fulfillment of the \\ requirements for the degree of \\ Master of Science in Aeronautics and Astronautics
}

University of Washington

2019

Committee:

Carl Knowlen

Mitsuru Kurosaka

Program Authorized to Offer Degree:

Aeronautics and Astronautics 
C Copyright 2019

Malik R. Washington 


\title{
University of Washington
}

\begin{abstract}
Radial Injector Mixing Effects on Detonation Zone Position in Rotating Detonation Engine
\end{abstract}

Malik R. Washington

Chair of the Supervisory Committee:

Dr. Carl Knowlen

Aeronautics and Astronautics

Radial propellant injection in the annular combustion chamber of a rotating detonation engine (RDE), done in a manner to promote maximum size scale of vortices, has proven to be effective for gaseous propellants. Experiments with an RDE having a 154-mm-diameter annular combustion chamber have explored the dependence of detonation zone location on propellant mass flux using gaseous methane and oxygen. Computational fluid dynamic simulations of the non-reacting flows of these experiments estimate that the degree of propellant mixedness at the leading edge of the detonation zone must be between approximately 0.5 and 1.5 standard deviations from stoichiometric. This computational tool was also used to examine the mixing of gases that were radially injected into a smaller RDE having a 76-mm-diameter annular combustor. The smaller RDE is designed for parametric studies of injector configurations, which 
may or may not be configured for radial injection. The results of this study indicate that staggered radial injectors lead to more rapid mixing compared to impinging radial injectors in annular ducts and that decreasing mass flux reduces the mixing length. 


\section{TABLE OF CONTENTS}

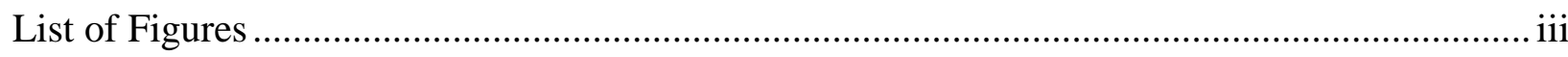

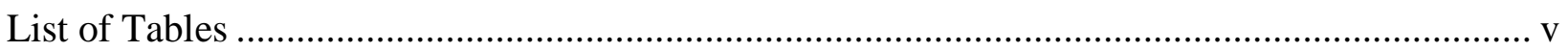

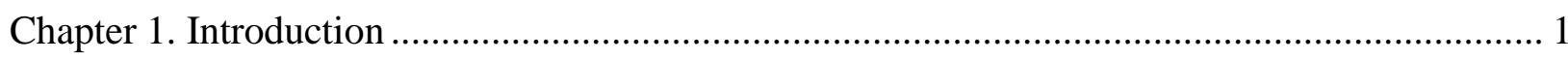

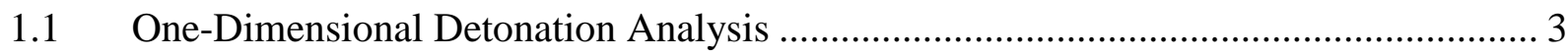

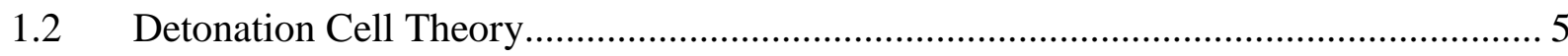

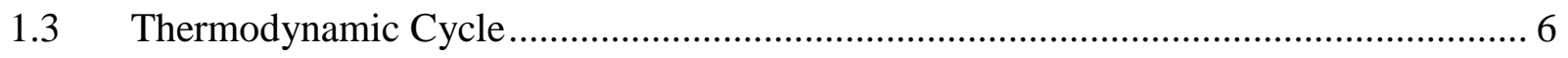

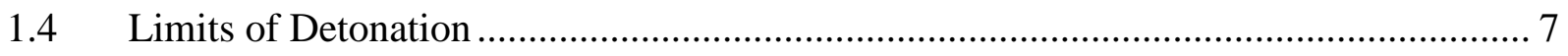

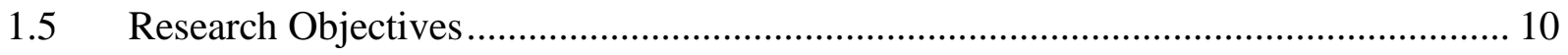

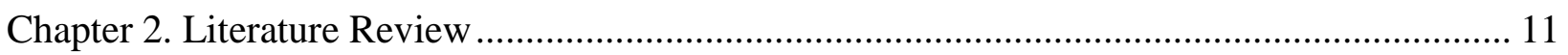

2.1 Pulse Detonation Engine Research .......................................................................... 11

2.2 Analytical Rotating Detonation Engine Research ………………........................... 12

2.3 Experimental Rotating Detonation Engine Research ................................................. 13

Chapter 3. Experimental Setup and Procedures............................................................................. 15

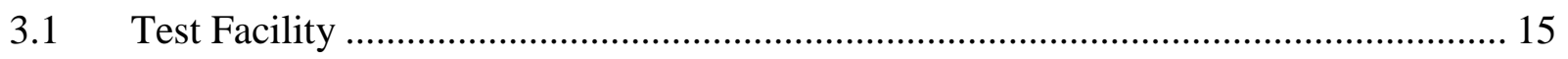

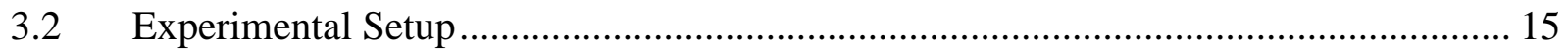

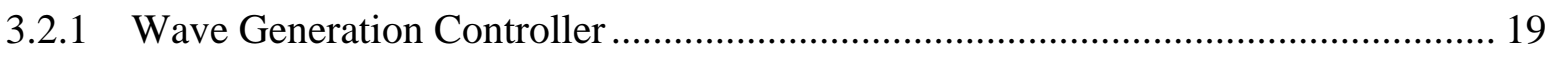

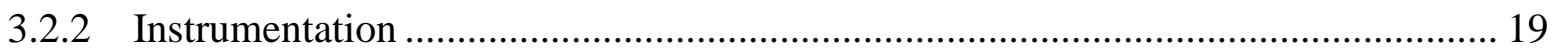

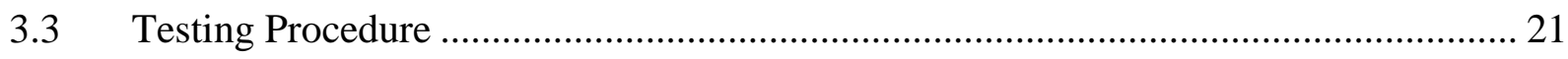

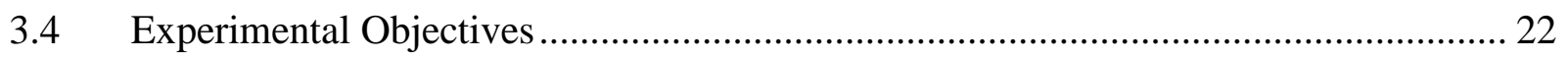


Chapter 4. Mixing in RDE

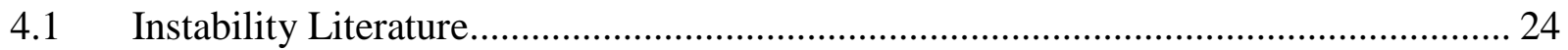

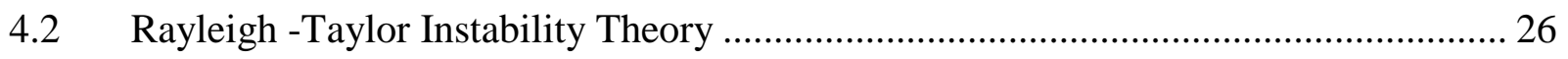

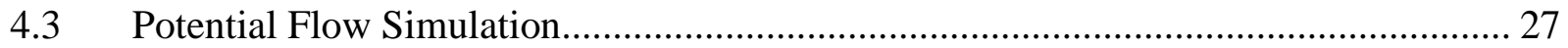

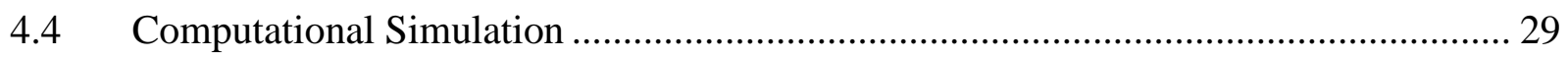

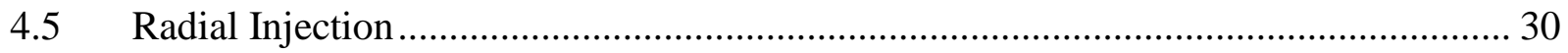

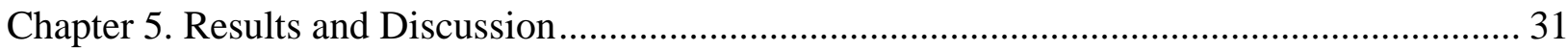

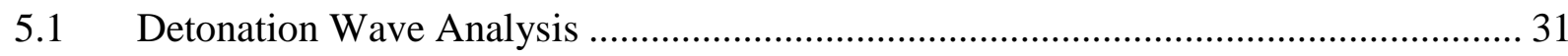

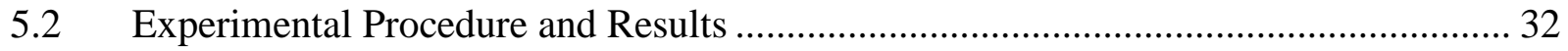

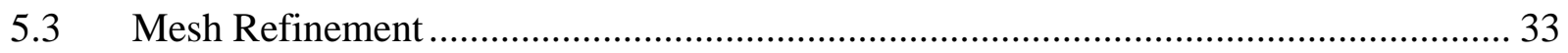

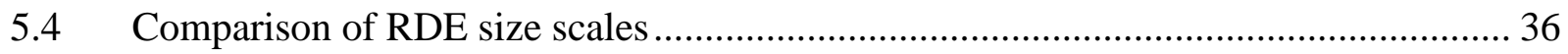

5.5 Injection Scheme Velocity Profile ............................................................... 39

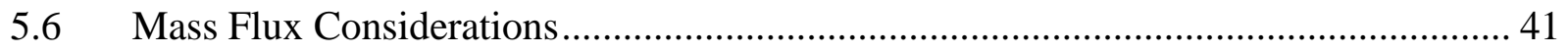

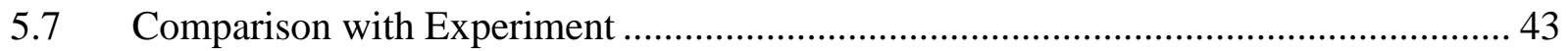

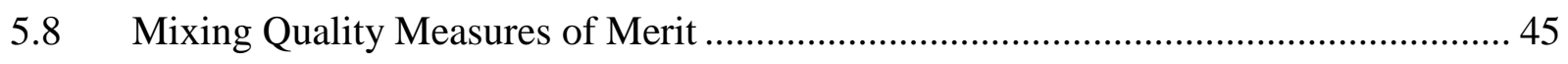

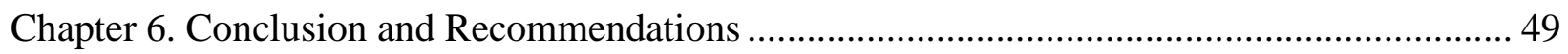

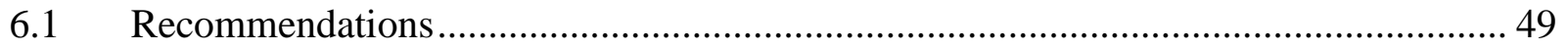

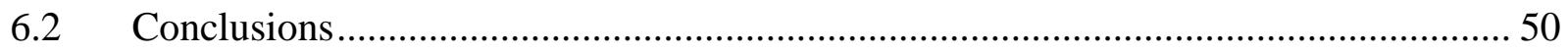

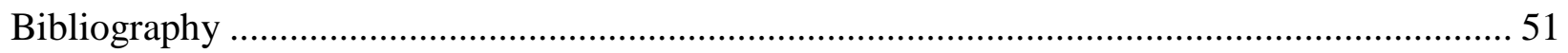

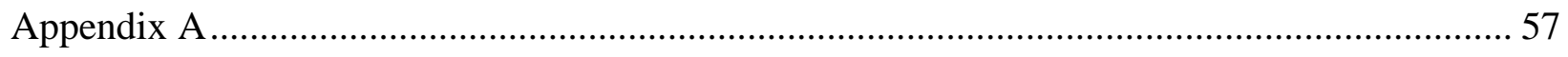

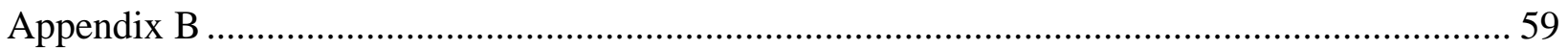




\section{LIST OF FIGURES}

Figure 1.1. Control volume problem for 1D detonation in constant area duct [4] ............ 3

Figure 1.2. Example of a unique solution to Rayleigh-Hugoniot relations [4]................. 4

Figure 1.3. Illustration of detonation cell structure [6] . .......................................... 5

Figure 1.4. T-s diagram for Humphrey, FJ, ZND thermodynamic cycles [8]. ................. 7

Figure 1.5. Ternary diagram for methane/oxygen/nitrogen [9]. ................................. 8

Figure 3.1. Floorplan of the SWR test facility. .................................................... 16

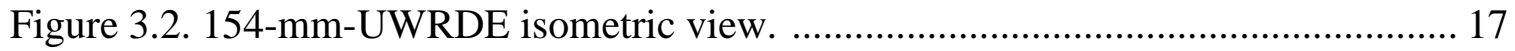

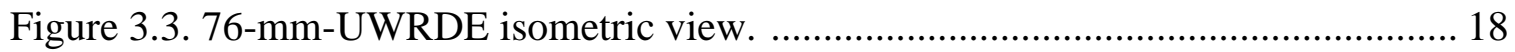

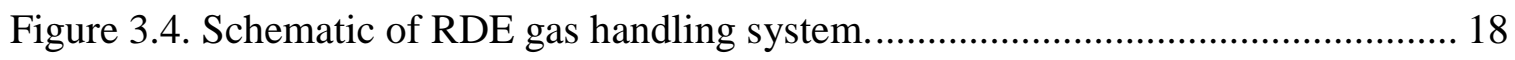

Figure 3.5. RDE test stand with WG spark boxes. ................................................ 19

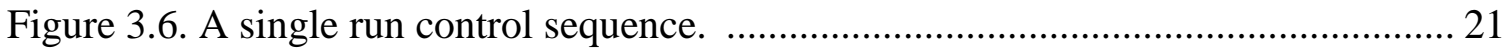

Figure 4.1. 2D illustration of the UWRDE staggered injection orifice scheme [25]....... 23

Figure 4.2. Full potential flow simulation of 154-mm-UWRDE mixing. ...................... 27

Figure 4.3. Potential flow simulation of 154-mm-UWRDE mixing. ............................ 29

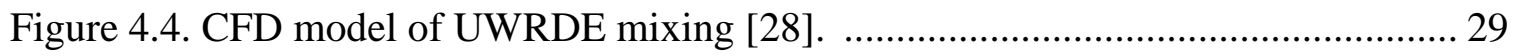

Figure 5.1. Peak temperature and location of thermocouple. ..................................... 33

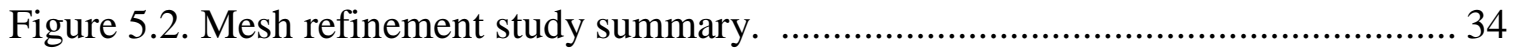

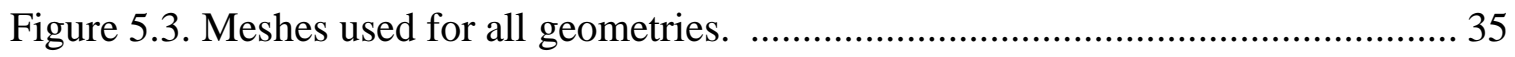

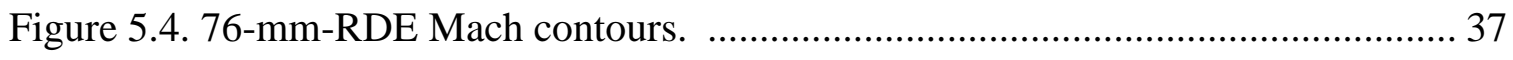

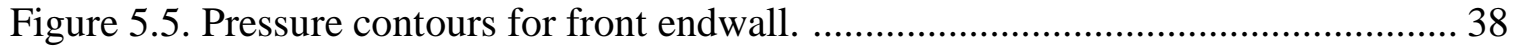

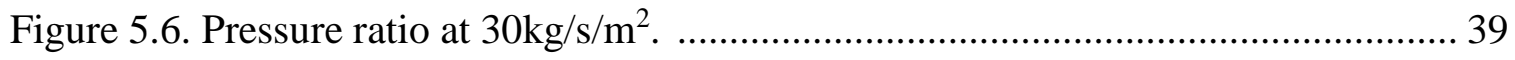

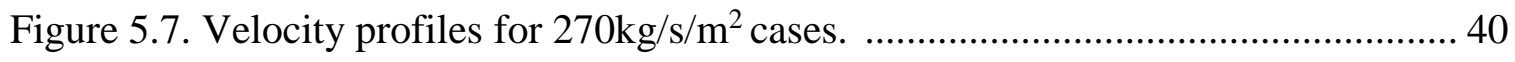

Figure 5.8. Velocity profiles overlaid on TKE contours. ........................................... 41

Figure 5.9. Velocity profiles of different mass flux cases. ....................................... 41

Figure 5.10. Equivalence ratio contours for 154-mm-RDE at varying mass flux. ......... 42

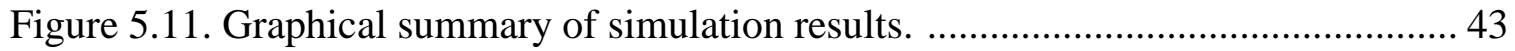

Figure 5.12. Equivalence ratio contour for $76-\mathrm{mm}-\mathrm{RDE}$ at $270 \mathrm{~kg} / \mathrm{s} / \mathrm{m}^{2}$. ................... 44 
Figure 5.13. Graphical summary of simulation results with trendline.

Figure 5.14. TKE contour for 76-mm-RDE. ........................................................ 48

Figure B.1. Pressure contours for $76-\mathrm{mm}-\mathrm{RDE}$ at $30 \mathrm{~kg} / \mathrm{s} / \mathrm{m}^{2}$. ............................... 59

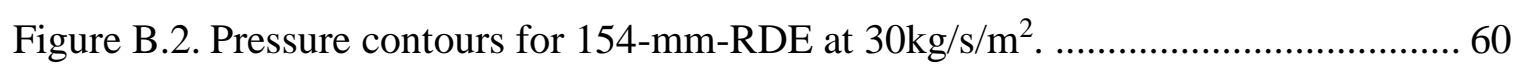

Figure B.3. Velocity profiles and overlaid TKE contours for 154-mm-RDE. ................. 62

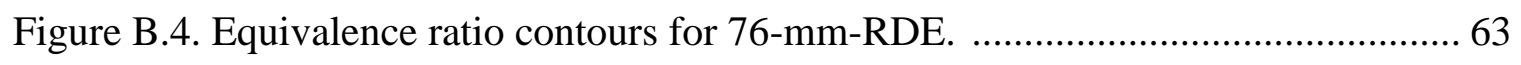




\section{LIST OF TABLES}

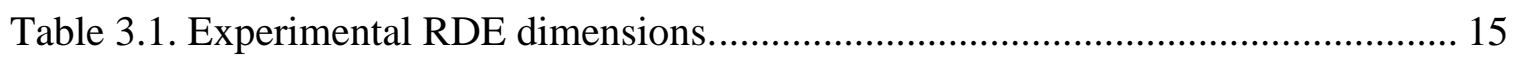

Table 3.2. Instrumentation probe location summary for UWRDE. ............................. 20

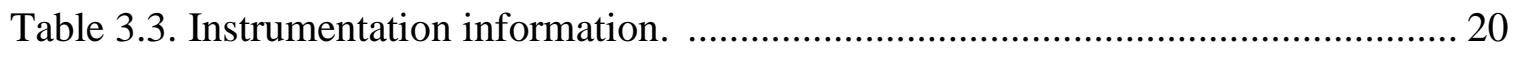

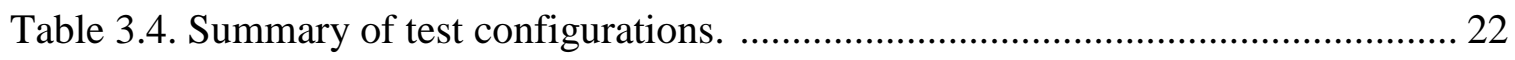

Table 5.1. Comparison of mixing measures of merit. ............................................. 46

Table 5.2. Comparison of mixing measures of merit with decay rate. ......................... 47

Table 6.1. Summary of detonation zone location results. ........................................ 49

Table B.1. Average pressure summary for $76-\mathrm{mm}-\mathrm{RDE}$ at $30 \mathrm{~kg} / \mathrm{s} / \mathrm{m}^{2}$. ......................... 61

Table B.2. Average pressure summary for $154-\mathrm{mm}-\mathrm{RDE}$ at $30 \mathrm{~kg} / \mathrm{s} / \mathrm{m}^{2}$. ................... 61 
This page was intentionally left blank 


\section{ACKNOWLEDGEMENTS}

The author is very appreciative of the financial support provided to this project by the Office of Naval Research (ONR) and Air Force Office of Scientific Research (AFOSR), for the productive climate created by the William E. Boeing Department of Aeronautics and Astronautics. He would like to thank his research advisors Carl Knowlen and Mitsuru Kurosaka for allowing him to join the University of Washington Rotating Detonation Engine (UWRDE) team. The members of the team-James Koch, Lien Chang, Daniel Méndez, Kevin Chau, Aaron Goldfogel, Chinmay Upadhye, Jeremy Nguyen, Jared Smythe, and Andrew Jacob-helped with gathering ideas, testing, and providing general support; they were invaluable in this endeavor. The outside expertise in the Aeronautics and Astronautics department from Pablo Posada and Abhiram Aithal in computational fluid dynamics (CFD) was extremely helpful. Additionally, he would like to thank Professor Alex Mamishev from the University of Washington Electrical Engineering for maintaining a Word file to use to format the following document. 
This page was intentionally left blank

viii 


\section{DEDICATION}

"Suck, Squeeze, Bang, Blow"

"Big whirls have little whirls,

That feed on their velocity;

And little whirls have lesser whirls,

And so on to viscosity."

-- Lewis Fry Richardson 
This page was intentionally left blank 


\section{Chapter 1. INTRODUCTION}

A rotating detonation engine $(\mathrm{RDE})$ is a propulsion system that utilizes detonation through a circular annulus channel to provide continuous quasi-steady thrust. It is the forefront of detonation engine research. The predecessor of the RDE is a pulse detonation engine (PDE), which provides intermittent thrust at a lower operating frequency in a longer, circular duct. The PDE is longer due to the distance for the deflagration-to-detonation transition (DDT) process. The operating frequency is lower and provides intermittent thrust due the time required to wait before injecting more reactants between each detonation. Succinctly, the PDE is inferior due to restrictions in size, cyclic frequency, mass flow rate, and thrust production [1]. These differences have shifted research to focus on RDE development due to greater applicability and benefits.

Detonation engine research has piqued interest for use in aircraft and space propulsion systems due to advantages over conventional deflagration combustion devices. These advantages include high thermal efficiency, high specific impulse, mechanical simplicity, and the ability to reach supersonic velocity without the use of a converging-diverging nozzle [2][3]. Detonation based propulsion became more practical through the development of compact, lightweight devices such as the PDE and RDE.

The potential benefits of pressure gain combustion with a rotating detonation engine (RDE) and the corresponding simplification of system designs for both air-breathing and rocket propulsion applications are significant, leading to the rapid expansion of research in this field $[1][4][5][6][7][8]$. Analytical and computational fluid dynamics (CFD) models have been developed to examine flow field characteristics within the annular combustor of an RDE and to predict thrust and specific impulse [9][10][11][12]. Simulations of annular combustors of RDEs 
with axially injected, premixed hydrogen/oxygen propellant show passing detonation wave fronts that propagate near the Chapman-Jouguet (CJ) speed [13][14]. Furthermore, analytical parametric cycle analyses of RDE performance as an ejector ramjet and two-stage low earth orbit rocket indicated that they had higher specific thrust and specific impulse than regular rockets [15]. For in-space propulsion applications, studies have shown that RDE propulsion offers many potential benefits over conventional rockets [16]. Understanding the intricacies of the internal fluid dynamics of RDE combustion chambers is key to realizing the benefits of RDE technology and the focus of the research presented here.

One topic of immediate interest is the means for propellant injection influences on the mixing process in annular combustors and the location of the detonation zone. Efficient mixing allows the detonation zone to move closer to the propellant injectors, which enables the use of shorter, more compact RDE combustion chambers. The conventional approach for propellant injection in an RDE utilizes many tiny orifices to encourage rapid mixing. Furthermore, the propellants are usually either co-injected axially or have one component injected radially and one axially. Experimental programs utilizing this propellant injection philosophy have all been successful in establishing RDE operation and are generating significant data over a wide range of operating conditions [16][17][18]. It has been suggested that the lower than predicted CJ speeds observed in these experiments is due to incomplete mixing; yet, the details of the respective mixing processes remain to be explored [19].

Another approach to mixing in a confined duct is to radially inject both propellant components. Previous studies have shown that the fine-scale motions, while ultimately responsible for the mixing at the molecular level, are not the rate-limiting process [20][21]. Thus, faster mixing is enhanced by investing the energy of the jets in the large-scale motions, which are maximized by 
radial injection [22]. By allowing the radial jets to impinge on the far wall, vortices are generated with the size scale of the annular channel height. In this process, the bulk of the propellants rapidly mix after at least one revolution of the vortices. The details of this mixing process and its consequences for RDE operation are the primary focus of this research.

\subsection{ONE-DimENSIONAL DETONATION ANALYSIS}

The detonation-driven combustion is the fundamental concept which provides the advantages over the deflagration-driven combustion in conventional engines. A detonation is a combustion process with a supersonic front. To understand detonation theory, a simplified 1D model is sufficient. Figure 1.1 shows the detonation problem setup.

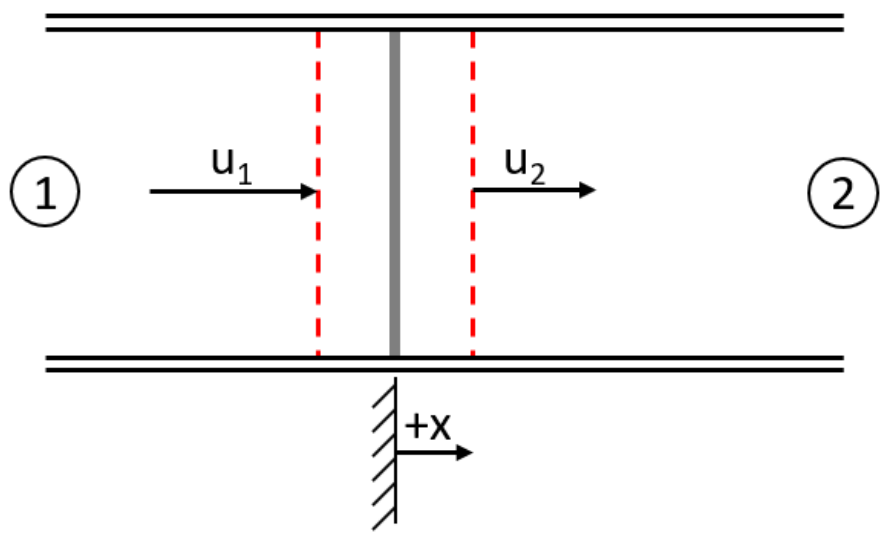

Figure 1.1 Control volume problem for 1D detonation in constant area duct [23].

The simplified model assumes 1D, adiabatic, inviscid, constant area flow with negligible body forces, and ideal and calorically perfect gases. The problem posed fixed the reference frame to the leading shock. Simplified conservation of mass (Eqn. 1), momentum (Eqn. 2), and energy (Eqn. 3) are given below:

$$
\begin{gathered}
\rho_{1} u_{1}=\rho_{2} u_{2} \\
p_{1}+\rho_{1} u_{1}^{2}=p_{2}+\rho_{2} u_{2}^{2}
\end{gathered}
$$




$$
h_{1}+\frac{u_{1}^{2}}{2}+q=h_{2}+\frac{u_{2}^{2}}{2}
$$

The subscripts 1 and 2 correspond to upstream and downstream of the leading detonation shock depicted in Fig. 1.1 [23].

Rayleigh lines are used to show the states that satisfy the conservation of mass and momentum. The Rayleigh line relation (Eqn. 4) is obtained by substituting Eqn. 1 into Eqn. 2.

$$
p_{2}-p_{1}=u_{1}^{2} \rho_{1}^{2}\left(\frac{1}{\rho_{1}}-\frac{1}{\rho_{2}}\right)=\frac{u_{1}^{2}}{v_{1}^{2}}\left(v_{1}-v_{2}\right)
$$

A Hugoniot curve satisfies conservation of mass, momentum, and energy. The Hugoniot (Eqn. 5) is obtained by substituting the Rayleigh relation (Eqn. 4) into the energy equation (Eqn. 3).

$$
h_{2}-h_{1}=\left(p_{2}-p_{1}\right) \frac{v_{1}+v_{2}}{2}-q
$$

The solution to satisfying the detonation jump conditions are the intersection of the Rayleigh line and Hugoniot curve [23]. Although there are mathematically two solutions, it has been shown that only one solution is physically possible. The detonation wave travels at a steady minimum velocity known as the Chapman-Jouguet (CJ) velocity. Figure 1.2 will be used to further explain the solution.

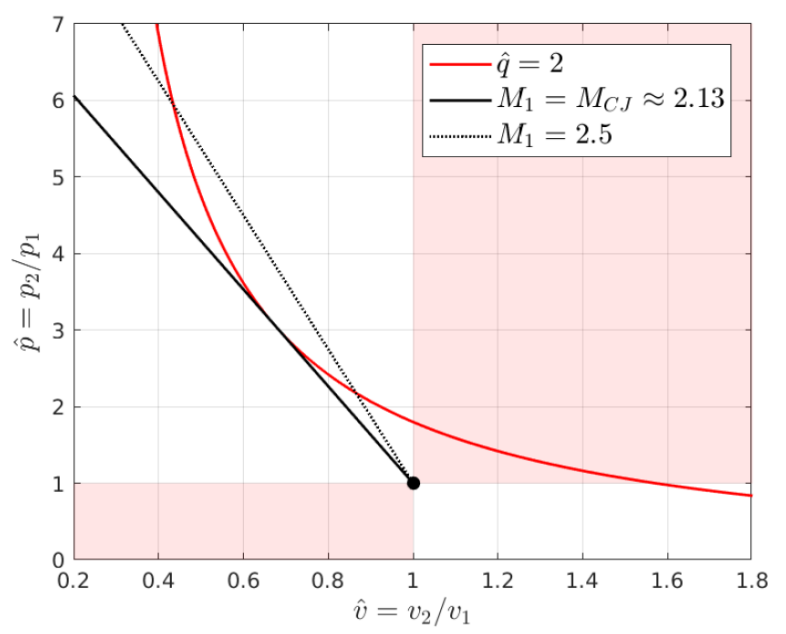

Figure 1.2 Example of a unique solution to Rayleigh-Hugoniot relations [23]. 
For a given heat release value, $\hat{q}=\left(\rho_{1} / p_{1}\right) q$, the incoming Mach number will be driven towards the minimum velocity found at the line tangent to the Rayleigh line and Hugoniot curve [23]. The heat release value is based on the chemistry of the detonation reactants. In detonation experiments, the incoming Mach number is designed to be higher than the CJ velocity because a lower Mach number will never be driven to the higher stable CJ velocity.

\subsection{Detonation Cell Theory}

The actual detonation structures in an RDE were thought to be important to understand because they were suspected to play limiting roles in the operation envelope and is important in understanding the internal detonation dynamics [24]. However, detonation cell size is strongly dependent on geometry. Figure 1.3 shows a detonation wave structure.

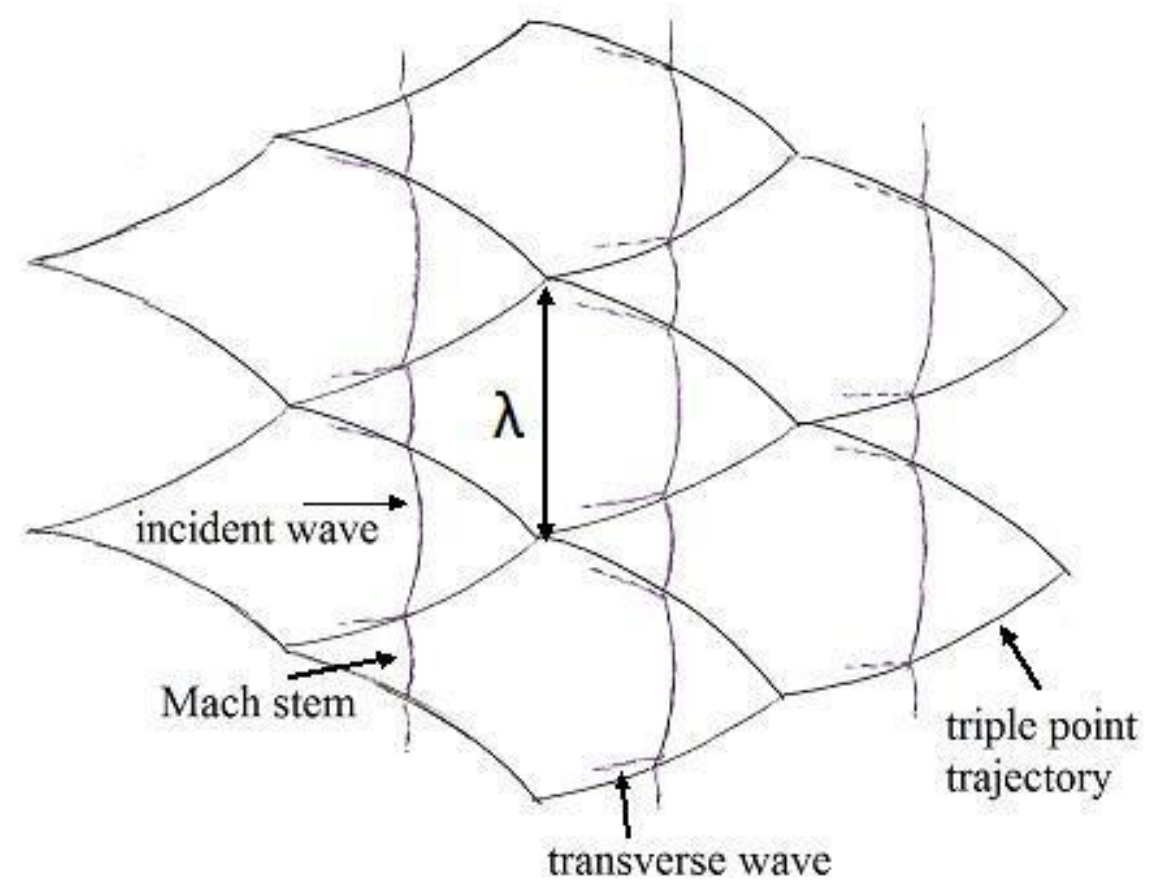

Figure 1.3 Illustration of detonation cell structure [25].

The detonation cell size, $\lambda$, is largely dependent on the detonation reactants. The cell size is determined by the spacing between the transverse waves. The transverse waves periodically collide 
or intersect with the leading shock wave to form triple points. The triple point phenomenon behind the repeated cellular pattern shown in Fig. 1.3. The triple point is the intersection of the Mach stem, incident wave, and transverse wave. The Mach stem and incident wave are the respective strong and weak parts of the shockwave [24].

The cell size for specific reactants were experimentally determined by analyzing smoked aluminum foils and frequency of pressure fluctuations. The cell size relation with RDE were suspected to describe the minimum annulus gap where detonation can occur. For an equivalence ratio of one, methane/air cell size is approximately $45.5 \mathrm{~cm} \mathrm{[26].}$

\subsection{THERMODYNAMIC CYCLE}

Although there has been some dispute on the proper thermodynamic model necessary to represent an RDE, the Zel'dovich-von Neumann-Doring (ZND) model currently most accurately represents the thermodynamic processes in the system. The ZND pressure-gain combustor thermodynamic cycle analysis is more appropriate than the Humphrey constant volume combustor thermodynamic cycle analysis and Fickett-Jacobs (FJ) cycle because the Humphrey model is not fully applicable to a detonation and the FJ cycle assumes instantaneous heat release [27]. Figure 1.4 shows a comparison of three different thermodynamic cycles for stoichiometric hydrogen/oxygen mixtures initially at standard temperatures and pressures.

Figure 1.4 shows the difference between the ZND and FJ model by how the heat addition process moves along a Rayleigh line. In terms of efficiency - the Humphrey, FJ, and ZND models show the RDE efficiency increase; the ZND model shows the RDE is more efficient than the FJ and Humphrey models [27]. Although the model was designed for a PDE, the thermodynamic cycles between the RDE and PDE are relatively similar. The cycle that most accurately represents a PDE will more accurately represent an RDE. 


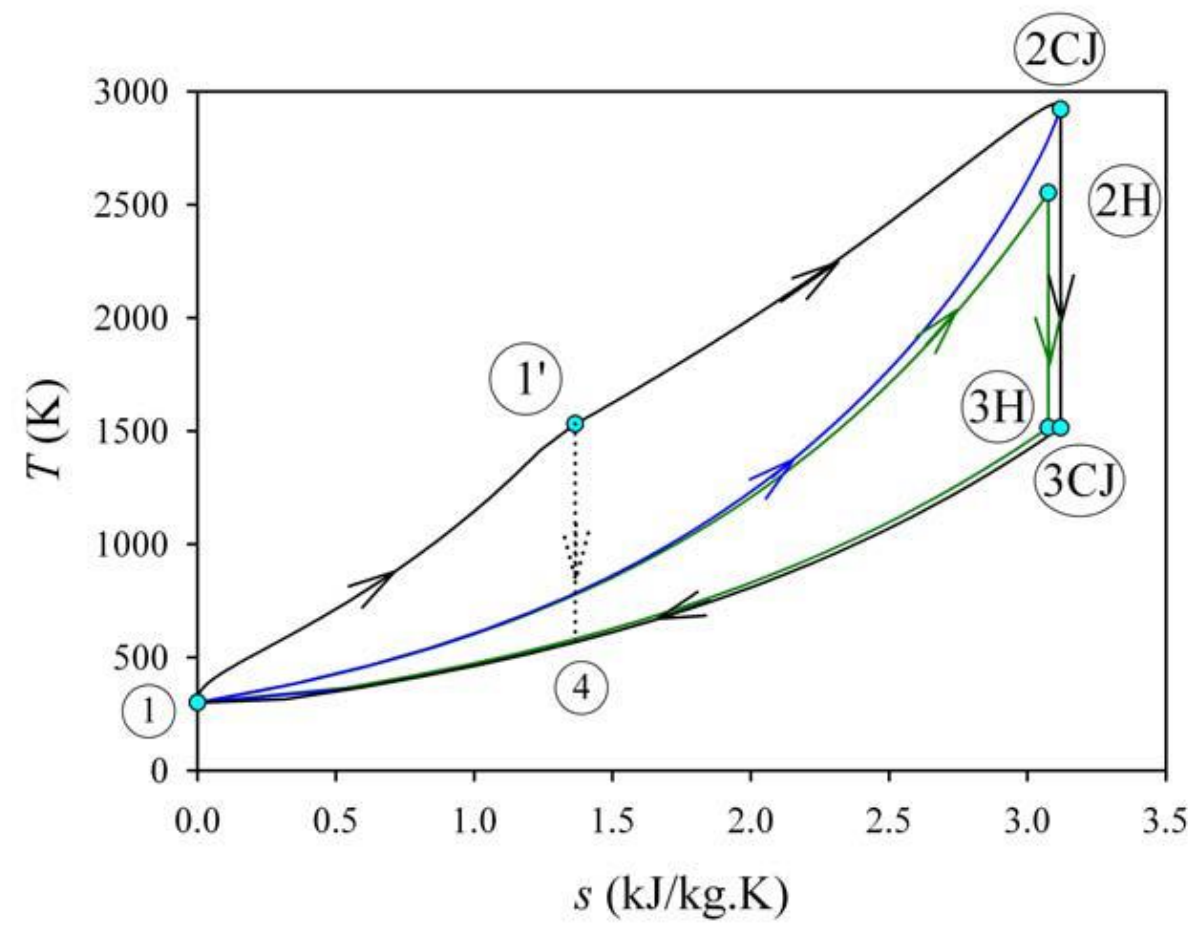

Figure 1.4 T-s diagram for Humphrey (green), FJ (blue), ZND (black) thermodynamic cycles for stoichiometric hydrogen/oxygen [27].

\subsection{LIMITS OF DETONATION}

A flammable mixture has the potential to detonate when subjected to a particular concentration, direction of flame propagation, pressure, and temperature. The limit where the mixture will combust where the fuel concentration is more dilute is known as the lower limit or combustiblelean limit. The other extreme is known as the upper limit or the combustible-rich limit [28].

The Bureau of Mines are an accepted standard for flammability mixtures. For a premixed mixture of methane/oxygen/nitrogen, its flammability limits can be represented on a ternary diagram like that shown in Fig. 1.5, which uses a thick line to depict the flammability envelope bounded by upper and lower flammability limits at atmospheric pressure and $26^{\circ} \mathrm{C}$ [28]. For methane/air mixtures, the flammability limits are 5\% to $15 \%$ (on a volume basis) under standard atmospheric conditions. In the absence of nitrogen, the far-left axis shows the rich and lean 
flammability limits of pure methane/oxygen mixtures are $60 \%$ and $5 \%$, respectively. The area enclosed by the flammable envelope boundary indicates mixture ratios that can sustain combustion, which is important for heat release processes that may arise downstream of detonation zone in an RDE. The detonable mixture range for any propellant, however, is always within the flammable limits. For example, at atmospheric pressure pure methane/oxygen will detonate at concentrations ranging from $10 \%$ to $40 \%$ [28] and methane/air detonable limits are $8 \%$ to $14.5 \%$ [29], as indicated Fig. 1.5. The full range of operability for an RDE and its relation to the detonable limits remain to be determined, but the RDE operability limits are expected to be within the flammable range of mixtures and be device dependent.

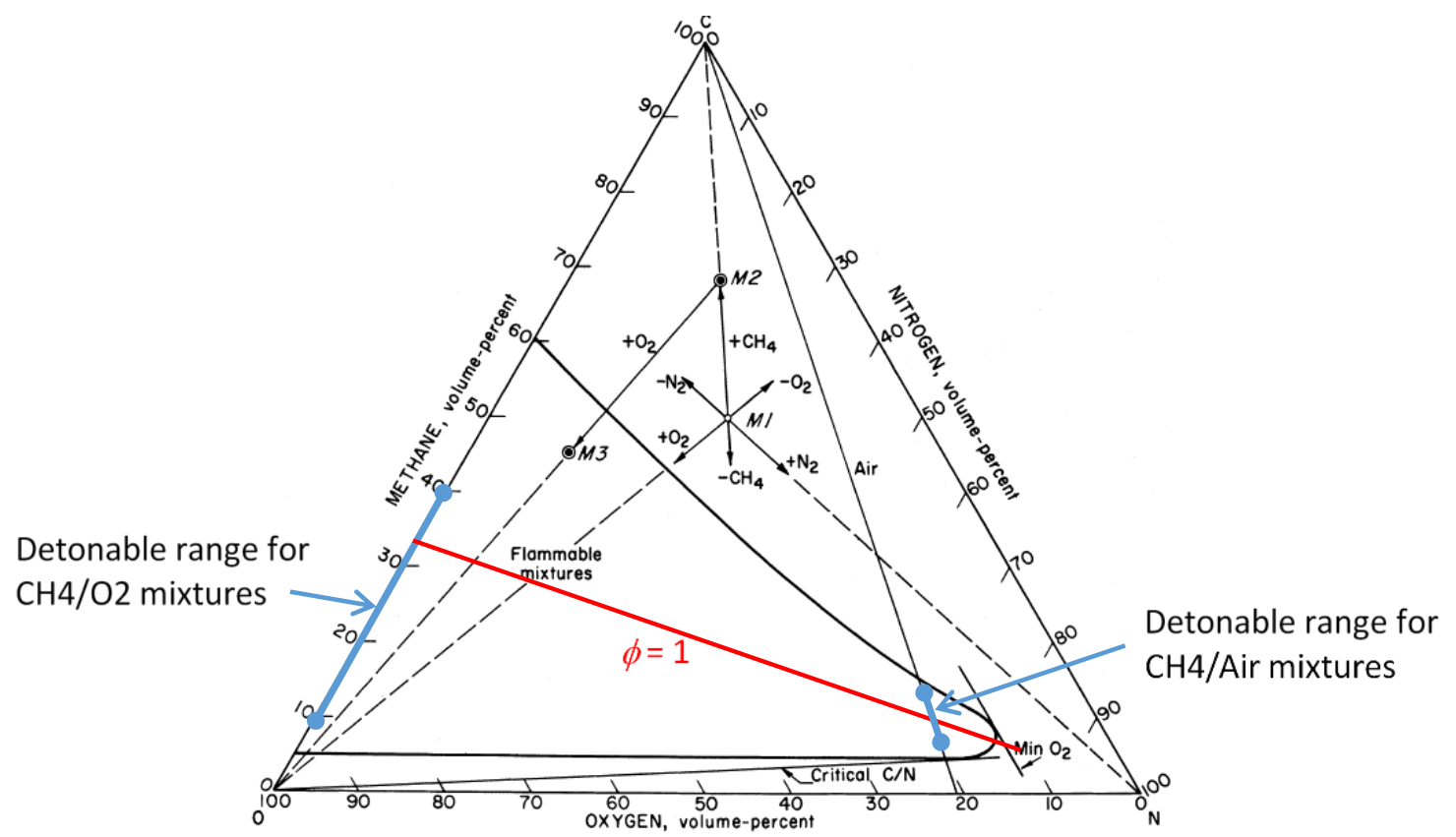

Figure 1.5 Ternary diagram for methane/oxygen/nitrogen at atmospheric pressure and $26^{\circ} \mathrm{C}$ [28].

A primary metric for the combustion properties of a reactive mixture is the fuel equivalence ratio, which is defined as the ratio of fuel/oxidizer to the stoichiometric fuel/oxidizer ratio as shown in Eqn. 6 below:

$$
\phi=\frac{m_{f u e l}}{m_{o x}} /\left[\frac{m_{f u e l}}{m_{o x}}\right] \text { stoichiometric }
$$


Equation 7 shows a stoichiometric chemical reaction between methane and oxygen gas mixtures. From Eqn. 7, the mole ratios for stoichiometric combustion is known and is used to calculate the molar fractions (Eqn. 8). The molar fractions for the rich (0.4) and lean (0.1) detonable limits are known from Fig. 1.5. Equation 9 shows the calculation to find the corresponding rich and lean equivalence ratio limits according to the flammability diagram (Fig. 1.5). The equivalence ratio limits of detonation combustion will occur somewhere between 0.15 and 1.8. The range for the 154-mm- and 76-mm-UWRDE must be experimentally determined.

$$
\begin{gathered}
\mathrm{CH}_{4}+2 \mathrm{O}_{2} \rightarrow \mathrm{CO}_{2}+2 \mathrm{H}_{2} \mathrm{O} \\
X_{i}=\frac{n_{i}}{\sum_{i} n_{i}}, X_{C H 4}=\frac{1}{3}, X_{O 2}=\frac{2}{3} \\
\phi_{\text {rich }}=\frac{X_{C H 4, \text { rich }}}{X_{C H 4}}=1.2, \phi_{\text {lean }}=\frac{X_{C H 4, \text { lean }}}{X_{C H 4}}=0.3
\end{gathered}
$$

Non-stoichiometric mixtures will have either excess fuel or excess oxidizer in its combustion products, depending on whether the equivalence ratio is greater or less than one, respectively. For pure methane/oxygen mixtures, the stoichiometric ratio is 0.5 or $33 \%$ on a volume basis, and for methane/air stoichiometric ratio is 0.105 or $9.5 \%$ of volume, as indicated by the red $\phi=1$ line in Fig. 1.5. Detonable mixtures of pure methane/oxygen reported in the Bureau of Mines, Bulletin 627 [28] had a range of $0.3<\phi<1.2$, however, the upper $\phi$ value represents only what data were available, not a limit. Stable operation of the RDE is most readily observed in mixtures that have equivalence ratios near $\phi=1$, although $\mathrm{RDE}$ operation has been demonstrated with methane/oxygen propellant having $0.25<\phi<2.5$ at the Air Force Research Laboratory facility [18] and with hydrogen/oxygen propellant having $0.8<\phi<1.8$ in the UW facility [30]. 


\subsection{RESEARCH OBJECTIVES}

Due to the limited understanding of the internal flow dynamics of an RDE, a small factor was investigated; the effect of mixing non-reacting reactants on detonation zone location was analyzed for a stoichiometrically injected methane/oxygen mixture. The following research was conducted to find whether or not there is a correlation with axial location of the detonation zone and the mixing. 


\section{Chapter 2. LITERATURE REVIEW}

Understanding what past research has been accomplished is essential to all future research. This section acknowledges the current understanding of RDE technology is essential building blocks for the research presented in the paper.

\subsection{Pulse Detonation Engine Research}

Research relating to PDE technology built the groundwork for RDE technology. Since the PDE is a simpler system and more closely resembles a shock or detonation tube, understanding the internal dynamics and workings is more manageable. Compared to the internal flow field in an RDE, the physics inside a shock tube are fairly well understood. This allowed the experimenters to calibrate and compare different kinds of pressure probes during detonation [31].

In terms of triggering detonation reactants, it was shown that properly triggered weak ignitors detonated premixed stoichiometric propane/oxygen/air and stoichiometric propane/air in detonation tubes. The distributed external energy source to reach activation energy for mixtures can be reached by successive triggering. This process uses less total initiation energy than the critical energy required from a single initiator [32]. This provided big insight on detonation schemes for PDEs. The UWRDE utilizes successive triggering to ignite reactants.

The investigation of thermal efficiency cycle for a PDE was developed and applied to obtain uninstalled performance characteristics - specific thrust, specific fuel consumption, and specific impulse. This provided an ideal upper limit of PDE performance. The PDE thermodynamic cycle was compared to the Humphrey and Brayton cycles [33].

The deflagration-to-detonation transition (DDT) process is important in detonation propulsion systems. In addition to analyzing mixing and stoichiometry to accelerate DDT speeds to reach 
transition, the experiment used a conventional Schelkin spiral to accelerate and overdrive detonation of hydrocarbon/air mixtures through a detonation tube within about $1.2 \mathrm{~m}$ for equivalence ratios of 0.9 to 1.3 . Additionally, the critical initiation energy for hydrocarbons are on the magnitude of $10^{5} \mathrm{~J}[34]$.

The end goal for propulsion systems are measured through performance factors (i.e. thrust). Analytical and experimental thrust measurement on PDE showed that equivalence ratio had direct correlation with thrust performance. For this particular engine, the best performance on this system was achieved with a slightly rich mixture, an equivalence ratio of 1.1 [35].

As mentioned in the Introduction section, obvious advantages of the RDE caused a shift in research away from PDE. However, the knowledge gained from PDE technology is closely related to RDE technology.

\subsection{Analytical Rotating Detonation Engine Research}

Due to the lack of understanding of internal RDE dynamics, analytical research is much more scarce compared to experimental RDE research. Understanding of physics behind a system can be shown through computational and analytical analysis that accurately reflect the physical system. Development of accurate computational models show a large step in understanding the internal physics in a system.

Computational models were developed based on solutions from previous algorithm solutions used on PDEs and conventional control volume analysis. This shows how closely the two detonation propulsion devices relate and the benefit of utilizing similar models to understand more complex systems. These models were used to better describe flow field within detonation channel and to predict thrust and specific impulse [36][12]. 
RDE computational fluid dynamics (CFD) models using detailed thermochemical processes for premixed hydrogen/oxygen injected axially in detonation channel showed passing detonation wave fronts are varied by geometry and total pressures can by varied by pressure of the reactive flow and stable detonation at CJ speeds [13][14].

Furthermore, analytical parametric cycle analysis of RDE performance as an ejector ramjet and two-stage low earth orbit rocket showed higher specific thrust and specific impulse than regular rockets [15]. RDE technology was shown to analytically perform better than conventional rockets in space propulsion systems.

Analytic models show performance and other deliverables that experimental research cannot obtain. Additionally, the models are much cheaper than conducting an experiment.

\subsection{EXPERIMENTAL ROTATING DETONATION ENGINE RESEARCH}

The first RDE experiment to characterize the structure of a detonation wave through a length of a detonation tube was in 1969. The authors obtained luminosity fields and locations of shocks with advancements in experimental photography [37].

It was nearly ten years later that steady detonation could be obtained with ethylene/oxygen/air mixtures. These detonations obeyed the CJ hypothesis for stable detonations, which provided a link between rotating detonation waves and acoustic instability [38].

It took thirty years after steady detonations were obtain with a high detonable hydrocarbon before it was shown that detonations can be sustained for nearly all gaseous and liquid hydrocarbon fuels [4]. This same paper showed the complex coupling of gasdynamics, thermodynamics, and geometry create large number of governing parameters for RDE operation-including other injection schemes. 
Within the past ten years there has been a significant increase in RDE research. There have been experiments to characterize RDE performance through detonation wave speed, steadiness of detonation wave, channel pressure, thrust, and mass flows. These experiments showed that although the largest engine operation occurred in the rich operating regime, high mass flow rates increased performance. However, higher oxygen flow rates were required to sustain detonation at these higher mass flow rates [39]. Higher mass flow rates decrease the detonation velocity due to the decreased mixing effectiveness [40].

Some other interesting relations showed that wave number is heavily influenced by the mass flow rates of the reactants [41]. Wave number is independent of upstream total pressure and annulus height [42]. Through varying pre-ignitor properties, there was a noticeable effect on initiation process and final stabilized detonation flow [43]. Different back pressures on the system have an effect on detonation and the speed of exhaust [44]. Inner chamber geometries have a significant impact on performance [45].

In terms of parametric studies, there have been an increased focus on analyzing what particular changes have on the RDE system. In order to determine the operation envelope of an ethylene/air RDE by varying mass flow rate, equivalence ratio, wave speed, and cell size [24]. A series of lower boundary operation envelope tests were conducted on a modular RDE that varied injection scheme and channel width by varying equivalence ratio and mass flow rates [17]. 


\section{Chapter 3. EXPERIMENTAL SETUP AND PROCEDURES}

This paper used two different engines developed at University of Washington (UW) for analysis. The 76-mm-RDE has a parametric injection scheme that will be used extensively to analyze mixing effects. The 154-mm-RDE has a set injection scheme which will be recreated as one of the parameters for the testing matrix. The unique features to the UWRDE and test facility are pure radial injection, spark plug array initiation, and the ability to change backpressure in the system.

\subsection{TEST FACILITY}

The experiment was performed in the High Enthalpy Flow Laboratory (HEFL) at the UW College of Engineering. The RDE system is accessed remotely from a control room. The control room is separated from the actual RDE system by a wall for safety reasons. The diluent gas and fuel bottles are located on the RDE system portion of the lab and the oxidizer gas bottles are located in the control room portion. The RDE exhaust and gases are isolated and ventilated after each run. Figure 3.1 shows a schematic of the RDE test facility.

\subsection{EXPERIMENTAL SETUP}

As discussed earlier, there are two engines used to for this experiment.

Table 3.1 Experimental RDE dimensions

\begin{tabular}{lcc}
\hline Engine Dimension Parameters & $154-\mathrm{mm} \mathrm{RDE}$ & $76-\mathrm{mm} \mathrm{RDE}$ \\
\hline Outer annulus diameter & $154 \mathrm{~mm}$ & $76.2 \mathrm{~mm}$ \\
Inner annulus diameter & $131.6 \mathrm{~mm}$ & $66.2 \mathrm{~mm}$ \\
Annular gap height & $11.2 \mathrm{~mm}$ & $5 \mathrm{~mm}$ \\
Combustor length & $177.8 \mathrm{~mm}$ & $163.5 \mathrm{~mm}$ \\
Fuel injection orifice diameter & $1.19 \mathrm{~mm}$ & $1.19 \mathrm{~mm}$ \\
Oxidizer injection orifice diameter & $1.78 \mathrm{~mm}$ & $1.98 \mathrm{~mm}$ \\
Number of orifices on each side & 24 & 36 \\
\hline
\end{tabular}




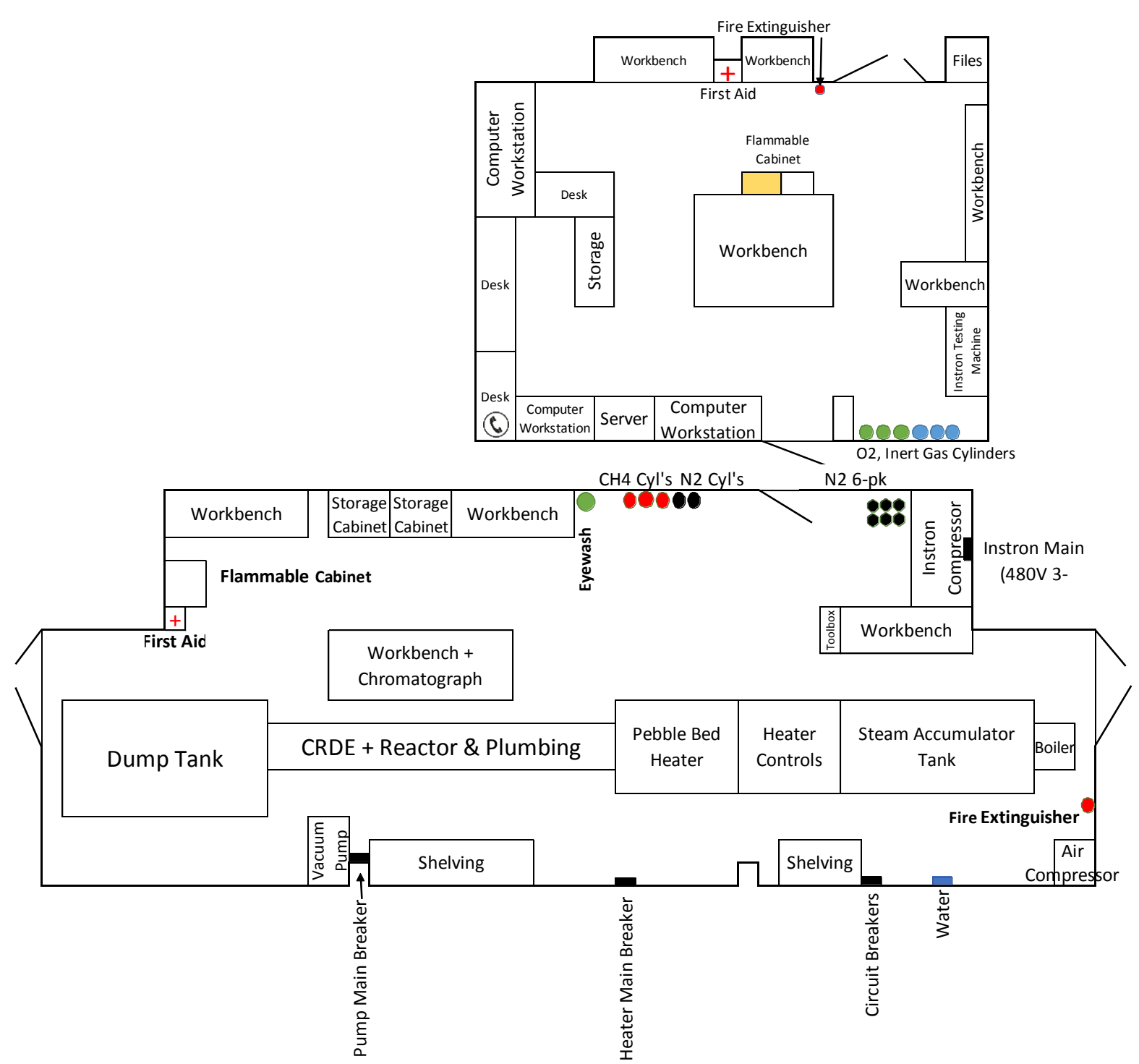

Figure 3.1 Floorplan of the HEFL test facility, control room (top) and experiment room (bottom).

Table 3.1 shows the geometric differences between the two engines.

The RDE experimental facility at the UW has been previously described [44][47][48][49].

To date, testing has only been carried out with an RDE having a combustor annulus outer diameter of $154 \mathrm{~mm}$ and channel height of $11.2 \mathrm{~mm}$ (see Table 3.1). This engine is fabricated from SS304 alloy. The outer sidewall of the combustor annulus had instrument ports that accommodated piezoelectric (PCB 112) and static pressure sensors (Omega PX319), as shown in Fig. 3.2 and listed in Table 3.4. Since the RDE currently tested in this facility utilize radial 
injection, the front endwall of the combustor annulus was instrumented for simultaneous pressure and temperature measurements. The RDE effluent in this facility is routed through a duct to a large dump tank that enables experimentation at backpressures ranging from sea level to vacuum conditions [50]. Although this particular experimental apparatus does not enable visual access to the exit of the combustor annulus for high-speed video, the spin velocities of the detonation waves, when they exist, were readily determined by FFT analysis of the highfrequency piezoelectric sensor data (sampled at $1.25 \mathrm{MHz}$ ). This approach has successfully determined the operability of the RDE over a wide range of equivalent ratios for both hydrogen/oxygen and methane/oxygen propellants [47][50].

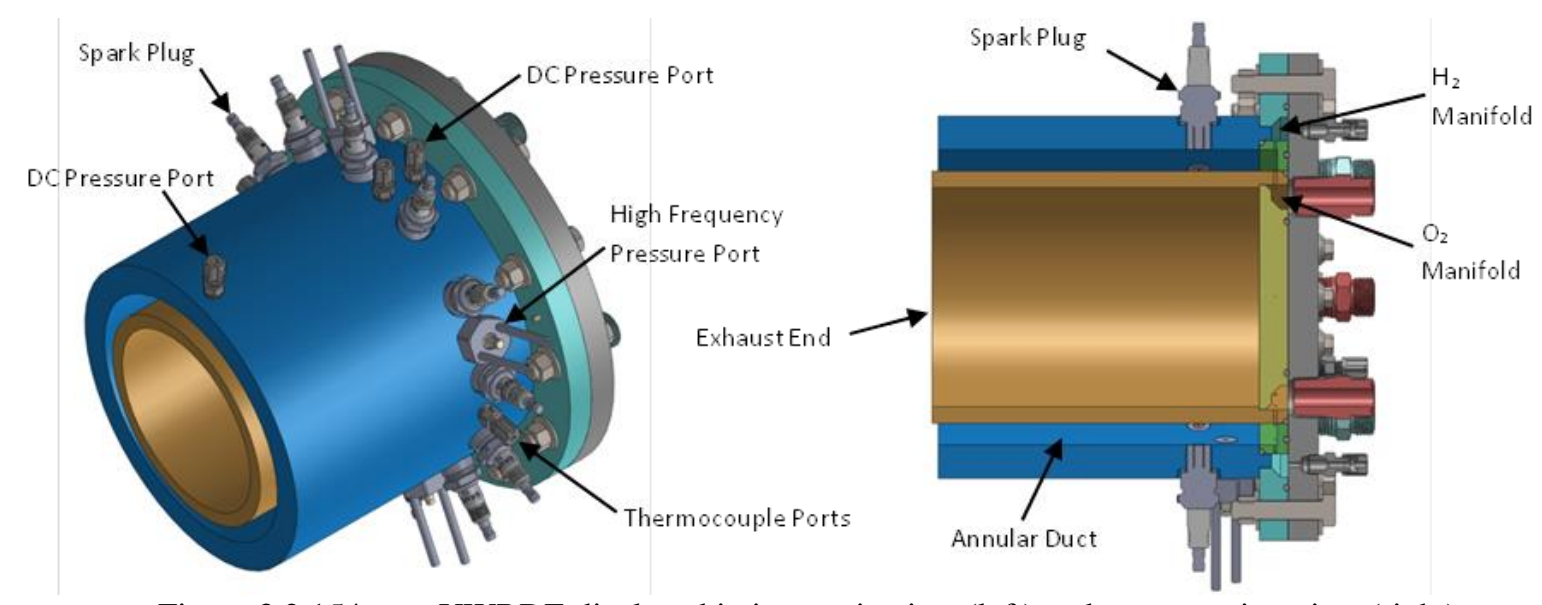

Figure 3.2 154-mm-UWRDE displayed in isometric view (left) and cross-section view (right).

The new RDE having a smaller annulus (76-mm-outer diameter), smaller channel height (5 $\mathrm{mm}$ ), and interchangeable injector orifices. The dimensions of this 76-mm-RDE, with injectors sized for stoichiometric methane/oxygen flow at equal manifold pressures, are compared with the 154-mm-RDE in Table 3.1. The new 76-mm-RDE is fabricated from both SS304 and C10100 alloy. The 76-mm-RDE design enables the insertion of different injector orifices that could be oriented in any direction, thereby enabling axial injection studies (Fig. 3.3) in addition to those with radial injection. The results from CFD simulations of the propellant injection processes for 
RDEs of similar design, but different size scales, enable examination of the respective flow fields and the influence of injector parameters on the rapidity of mixing in the annuli.

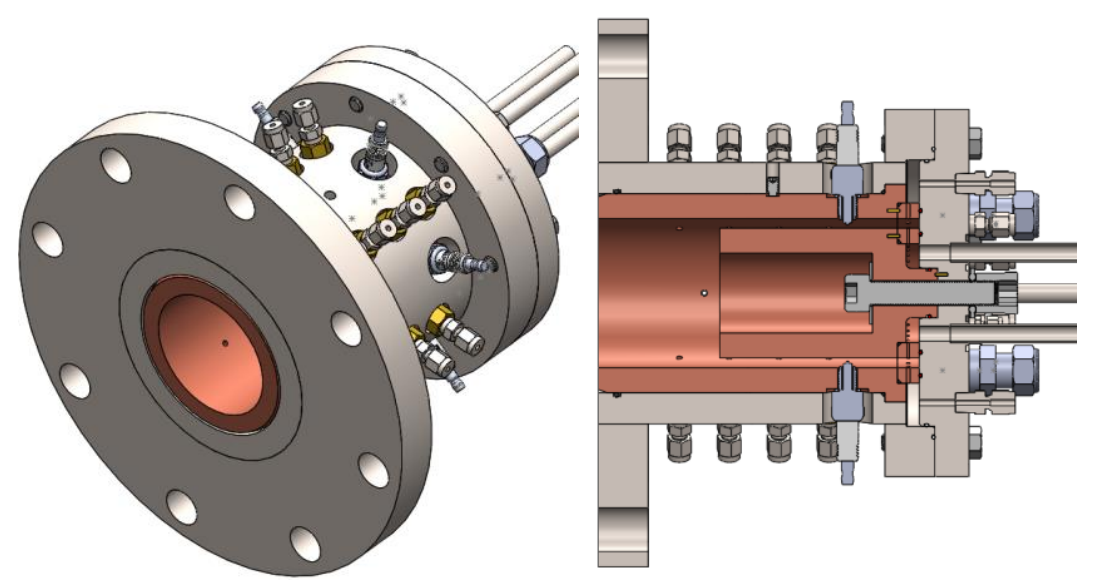

Figure 3.3 76-mm-UWRDE displayed in isometric view (left) and cross-section view (right).

In terms of gas handling, a generic schematic is depicted in Fig. 3.4. The gas handling system was recently updated for visual access, higher flow rates, and higher instrumentation density. Throughout the update this general schematic holds accurate.

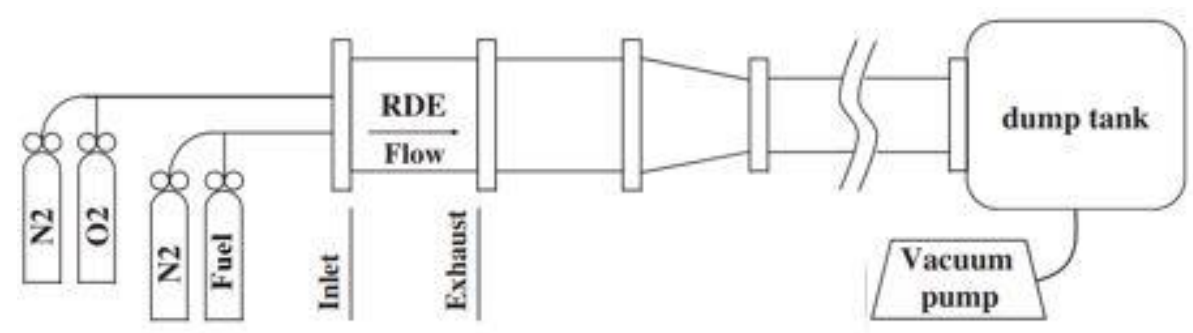

Figure 3.4 Schematic of RDE gas handling system.

The gas bottles are commercially purchased and were pressurized between $2500 \mathrm{kPa}$ and 18000 $\mathrm{kPa}$ throughout the experiment. The lower limit was established when the bottles were unable to feed the system a constant flow to achieve a particular mass flux and equivalence ratio. Spent gas is sent to an approximately 4 cubic meter dump tank and sent out of the facility through an isolated duct to the roof. 


\subsubsection{Wave Generation Controller}

The wave generator (WG) (Fig. 3.5) was designed by Prof. Tom Mattick to initiate the reactants in the UWRDE. The wave generation controller can vary the power produced by each spark plug, the frequency the spark plugs sequential firing, and the number of simultaneous sparks. The initial idea behind the WG was to control the number and direction of waves. However past research at UW showed no correlation in number of waves produced [48]. For this experiment only six of the 12 spark plugs in the array were utilized for testing and each spark plug was individually and sequentially fired. This detonation initiation method is a unique feature of the UWRDE.

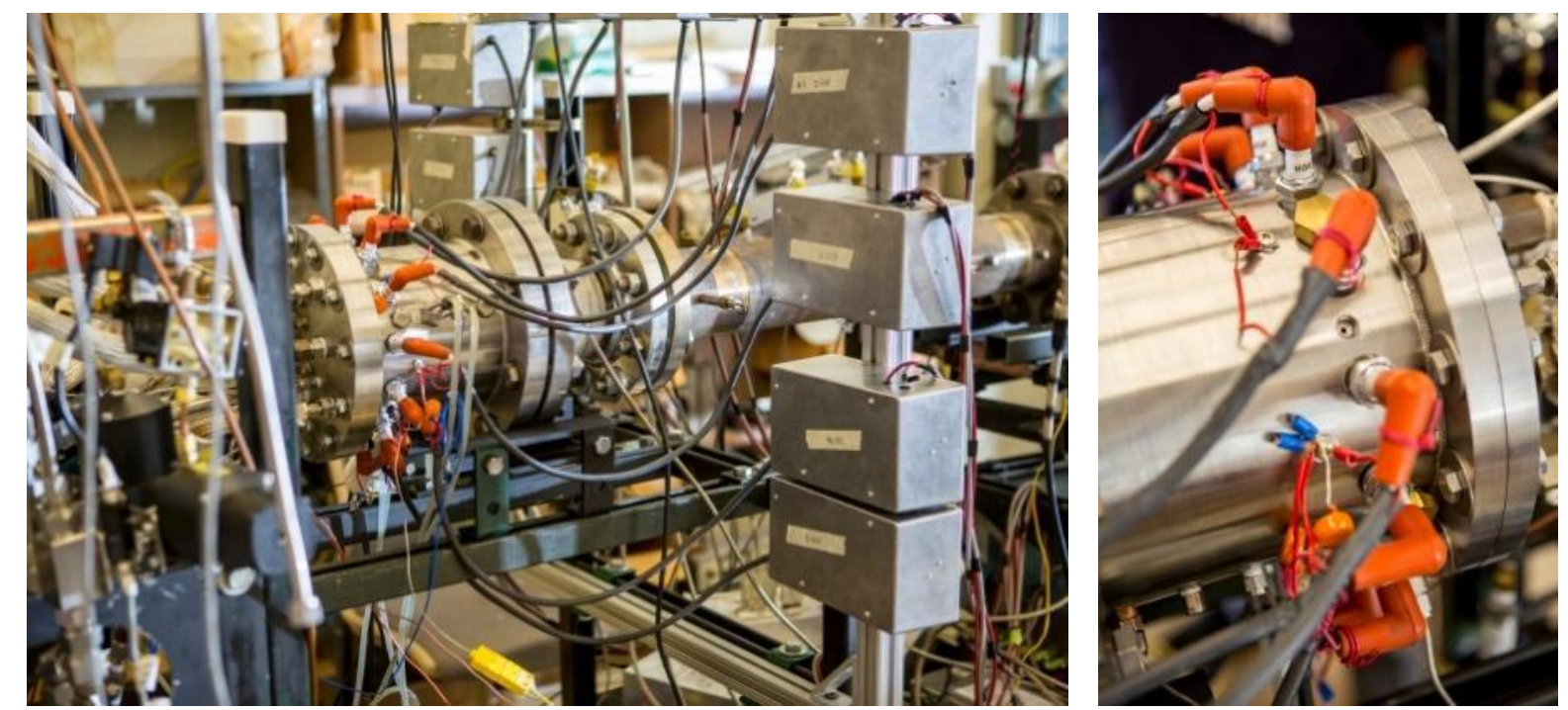

Figure 3.5 RDE test stand with WG spark boxes (left), close-up of WG spark plugs installed on RDE system (right).

\subsubsection{Instrumentation}

The instrumentation used for this particular experiment included thermocouples, capillary time averaged pressure (CTAP) probes, and high frequency PCB pressure probes, and ionization probes. These probes were used to identify the detonation zone position and characterize the system. Additional instrumentation ports are shown in Fig. 3.2 but were not utilized for this 
particular analysis. The locations of the instrumentation probes used for this experiment are summarized in Table 3.2.

Table 3.2 Instrumentation probe location summary for UWRDE.

\begin{tabular}{|c|c|c|c|c|}
\hline \multirow[b]{2}{*}{ Instrumentation Probe } & \multicolumn{2}{|c|}{ 154-mm-RDE } & \multicolumn{2}{|r|}{ 76-mm-RDE } \\
\hline & $\begin{array}{l}\text { Azimuthal } \\
\text { Position }\left[{ }^{\circ}\right]\end{array}$ & Axial Distance & $\begin{array}{l}\text { Azimuthal } \\
\text { Position }\left[{ }^{\circ}\right]\end{array}$ & Axial Distance \\
\hline Axial thermocouple & N/A & N/A & $30 ; 90$ & $\begin{array}{c}8.02,13.06,18.11,23.18 \\
5.43,10.47,15.53,20.61\end{array}$ \\
\hline Front wall thermocouple & 205 & -0.10 & $15,60,115$ & -1.27 \\
\hline Axial CTAP probes & $255 ; 270$ & $\begin{array}{c}7.82,10.67,13.53 ; \\
6.39,9.24,12.10\end{array}$ & $150 ; 210$ & $\begin{array}{r}8.02,13.06,18.11,23.18 \\
5.43,10.47,15.53,20.61\end{array}$ \\
\hline Front wall CTAP probes & 205 & -0.10 & 195, 295 & -1.27 \\
\hline PCB probes & $225 ; 285$ & $-0.10 ; 3.67$ & $195 ; 210$ & $\begin{array}{r}8.02,13.06,18.11,23.18 \\
5.43,10.47,15.53,20.61\end{array}$ \\
\hline Axial ionization probes & N/A & N/A & $270 ; 330$ & $\begin{array}{r}8.02,13.06,18.11,23.18 \\
5.43,10.47,15.53,20.61\end{array}$ \\
\hline
\end{tabular}

The axial distance is nondimensionalized to the annular gap height and is measured from the injection orifice plane of the system. The front wall thermocouple instrumentation port will be used to monitor the front wall temperature and perform front wall insertion tests to locate the detonation zone.

Table 3.3 shows manufacturer and model information for the select probes used for identifying the detonation zone position and other transducers used to identifying flow characteristics.

Table 3.3 Instrumentation information.

\begin{tabular}{lcc}
\hline Sensor & Manufacturer & Part No. \\
\hline High frequency pressure transducer & PCB Piezotronics & $112-$ A05, 113-B22 \\
Manifold pressure & Omega Engineering & PX319-1KG5V \\
Venturi meter pressure & Omega Engineering & PX319-500G5V \\
Combustor pressure & Omega Engineering & PX319-500G5V \\
Type K Thermocouple & Omega Engineering & TJ36-CASS \\
\hline
\end{tabular}


The low frequency measurements are sampled at $2 \mathrm{kHz}$ and the high frequency response measurements are sampled through the high-speed data acquisitions system (DAQ) at 1.25 MHz. In the past, the higher frequency measurements were used solely to characterize the wave spin speed. However, higher frequency measurements from multiple PCB transducers are planned as an alternate means to identify the detonation wave position.

\subsection{Testing Procedure}

Each experiment run was conducted according to specific procedure. First, the pre-run checklist (Appendix A) steps were followed and necessary information was recorded in an Excel spreadsheet. Throughout the experiments, the butterfly setting was set to 15 (no flow restriction between the dump tank and system) and the WG wave number is set to $\mathrm{N}=1$. The sparking and gas valve control sequence for all the runs is shown in Fig. 3.6.

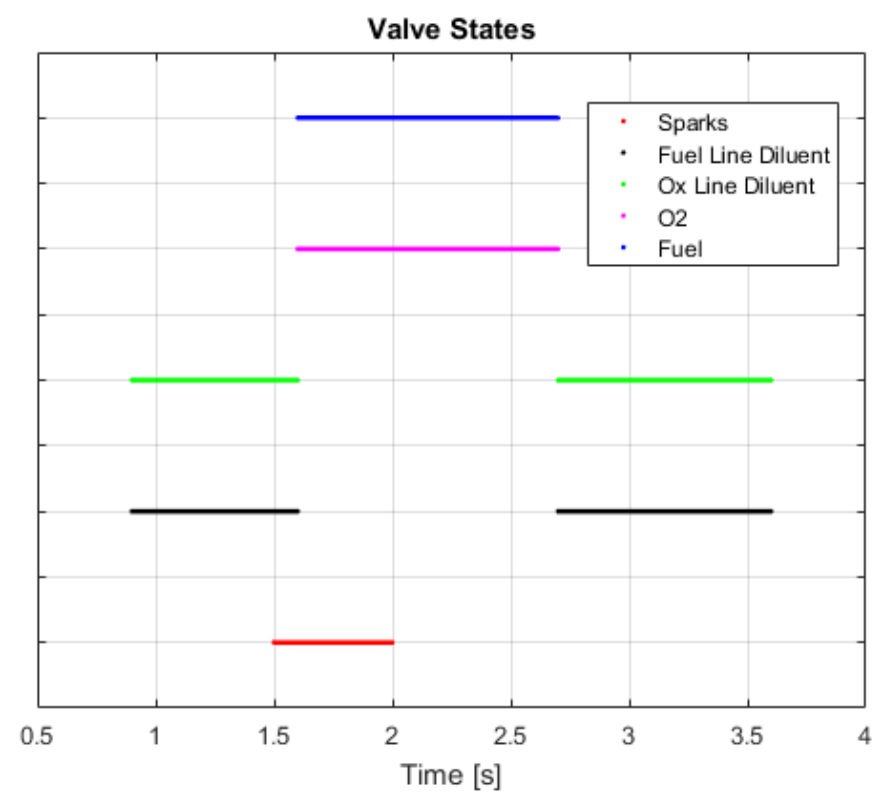

Figure 3.6 A single valve state control sequence. 


\subsection{EXPERIMENTAL OBJECTIVES}

The detonation zone location will be determined for the 154-mm-RDE for a stoichiometric methane/oxygen mixture. The same injection scheme with the same mass flux and a higher mass flux will be determine for the 76-mm-RDE for the same reactants. The 76-mm-RDE will be used to identify the detonation zone location for the same reactants at the same higher mass flux with an additional injection scheme. Table 3.4 summarizes the experiment.

Table 3.4 Summary of test configurations.

\begin{tabular}{cccccc}
\hline $\begin{array}{c}\text { Configuration } \\
\text { Designator }\end{array}$ & $\begin{array}{c}\text { RDE OD } \\
{[\mathrm{mm}]}\end{array}$ & $\begin{array}{c}\text { Injection } \\
\text { Geometry }\end{array}$ & $\begin{array}{c}\text { Equivalence } \\
\text { Ratio }\end{array}$ & $\begin{array}{c}\text { Backpressure } \\
{[\mathrm{kPa}]}\end{array}$ & $\begin{array}{c}\text { Mass Flux } \\
{\left[\mathrm{kg} / \mathrm{s} / \mathrm{m}^{2}\right]}\end{array}$ \\
\hline $1-1-1$ & 154 & staggered & 1.0 & 10 & 10 \\
$1-1-2$ & 154 & staggered & 1.0 & 10 & 20 \\
$1-1-3$ & 154 & staggered & 1.0 & 10 & 30 \\
$2-1-3$ & 76 & staggered & 1.0 & 10 & 30 \\
$2-1-4$ & 76 & staggered & 1.0 & 10 & 270 \\
$2-2-4$ & 76 & impinged & 1.0 & 10 & 270 \\
\hline
\end{tabular}

The first number in the configuration designator represents the 154-mm-UWRDE and 76-mm-

UWRDE. The second number represents the injection geometry. The last number represents the mass flux. The configuration designator will be referenced throughout the remainder of the report to identify different testing configurations. 


\section{Chapter 4. MIXING IN RDE}

The mixing scheme in an RDE plays an important role in the detonation process. Since the vast majority of models assume fuel and oxidizers are completely mixed, engine designs should strive to mix reactants as much as possible. Incomplete mixing degrades the accuracy and performance of designs and equivalence ratio calculations. The equivalence ratio denotes the fuel/oxidizer ratio to the stoichiometric fuel/oxidizer ratio (Eqn. 6). The equivalence ratio is important for characterizing the operation envelope of an RDE. It has been shown through experiments that detonation fails when the mixture is too rich or lean. Poor mixing impacts these operation envelopes and plays a large factor why different engines have different operating equivalence ratios. Efficient mixing blends reactants fully in relatively short amounts of time and space. Most RDEs introduce fuel and oxidizer through some combination of radial and axial directions and mix by impinging one on the other. The RDE examined here has a unique mixing scheme that involves the radial injection of both fuel and oxidizer, as shown in Fig. 4.1 [44][47][48][49]. The transverse injectors in this radial injection scheme are staggered to maximize the size of the vortex generated by jet impingement on the far wall. In the case shown here, fuel (red) is injected from orifices on

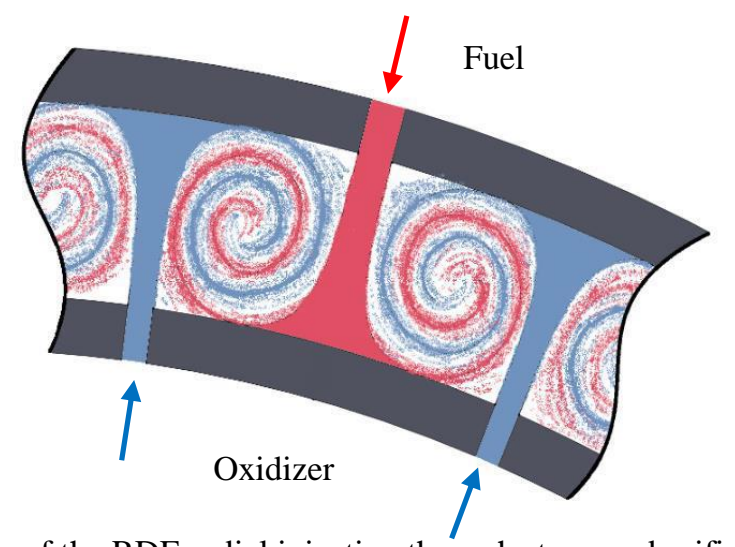

Figure 4.12D illustration of the RDE radial injection through staggered orifice in annular combustor [41]. 
the curved outer wall of the annulus and the oxidizer (blue) is injected from ports in the inner cylinder surface. The vortical motion of the two propellant components permeates throughout the volume, which, in principle, encourages bulk mixing of the flow within a few channel heights downstream of the injectors.

The transverse injectors in this radial injection scheme was designed with the idea that mixing would occur as quickly as possible. The circulation of the vorticity stirs the two fluids together as the fluid mixes further downstream. It has been shown that the magnitude of the concentration fluctuations declines as flow progresses further downstream with this injector geometry setup [53][54]. The radial injection scheme in the UWRDE allows mixing to occur further upstream which would allow for a shorter RDE system. As opposed to mixing by impinging oxidizer and fuel, the mixing occurs through vortical, turbulent mixing. To further investigate the exact mixing scheme depicted in Fig. 4.1, qualitative information can used to better understand the dynamics inside the UWRDE injection scheme. The literature discusses experimental, numerical, and analytical models on 2D and 3D Lamb-Oseen vortices, vortex rings, vortices and corresponding relationships with the Rayleigh-Taylor (RT) instability, Kelvin-Helmholtz instability, and mixing in general. The main mixing theory that the UWRDE capitalizes on is the RT instability.

\subsection{INSTABILITY LITERATURE}

Literature analyzes the Rayleigh-Taylor instability in different ways. The takeaways give different information that can be applied to the UWRDE.

An array of direct numerical simulations (DNS) for a 2D Lamb-Oseen vortex by varying internal core densities show that RT instabilities arise from the destabilizing effect of the centrifugal force on the radial flow density gradients. The instabilities were driven by density peaks which resulted in efficient mixing [55]. 
The RT instability appears in density gradients between a single and ambient fluid. Experimentally created vortex rings in a water tank were generated by forcing fluid through a small orifice like the RDE injection holes. It was shown through laser-induced fluorescence flow visualization technique that rapid mixing occurred through an increase in vortex ring diameter. The vortex ring vorticity began with a Gaussian distribution and, as the vortex ring rolled up into the core due to density gradients, the distribution was diverted partially to the vortex roll-up [56]. This shows that the instability arises as the fuel/oxidizer are injected, before impacting the wall.

In another experiment, the velocity flow field developments were extracted from particle image velocimetry of a liquid vortex ring impacting a surface. Although the research was geared towards understanding microbursts from density-driven downdrafts and low-altitude wind shear, it relates directly to the radial injection problem. As expected, the horizontal velocity instantly increases with surface contact. The experiments showed that after wall impact the unsteady adverse pressure gradients cause the flow to separate and roll up into secondary vortices [57]. This is the same phenomenon projected to be seen in the UWRDE injection scheme and in other RT instability problems. The interaction between the main vortex and the secondary vortices resulted in large spatial variations in velocity which will result in mixing.

In another DNS, a second, counter-rotating Lamb-Oseen vortex is added to simulate downwash created from aircraft wing tips. This applies less to the UWRDE problem because the simulation would require two fluids rotating in the same direction. However, this paper utilizes the superposition of potential flow models in the development of the DNS solutions [58]. Potential flow model assumptions assume irrotational flow, thus ignoring dominant features of the late stage vorticity field [59]. 
An experimental technique utilizing solid rocket motors studies the growth of RT instabilities in fluids. This experiment showed the density difference between the two fluids did not impact the penetration depth between the fluids. However, it did show that long wavelength perturbations increase instability [60]. This shows utilizing long wavelength perturbation to increase instability increases mixing, but it would prove to be difficult to implement in an injection scheme in an RDE.

Other literature discussed other instabilities that would affect mixing in UWRDE like the Kelvin-Helmholtz instability. The Kelvin-Helmholtz instability arises due to the density interface not being perfectly circular [59]. This kind of instability most likely arises in the UWRDE. However, it is insignificant compared to the instabilities due to density gradients. Although there are other types of vortex-induced instabilities, the RT instability plays the largest role in the UWRDE.

\subsection{RAYLEIGH -TAYLOR INSTABILITY THEORY}

The bottom line from the literature is that the RT instability promotes efficient mixing through turbulence [55][56][59][60]. The RT instability is fundamental for understanding the dynamics between fluids of different densities [59]. The theory shows that efficient mixing is a direct result of this RT instability which is why the UWRDE attempts to take advantage of the phenomenon. Figure 4.1 illustrates fuel (red) injected from an orifice on the outer diameter and the oxidizer (blue) injected from the inner diameter. The RT instability arises due to the differing densities between the fuel and oxidizer. There is a destabilizing effect of the centrifugal force on the flow density gradients [59].

The RT instability begins the moment, the fuel/oxidizer are injected from the holes. The density gradient between the air already in the system also contribute to creating instabilities in the fluids before they impact the wall [56]. The UWRDE injectors can be modeled to some degree 
of accuracy through potential flow theory as long as the model understands the limitations and inability to show the instabilities.

\subsection{Potential Flow Simulation}

Ideally, the vortical mixing from radial injection would be modeled in three dimensions by two vortex rings injected in opposite directions. The mixing must be analyzed in three dimensions to gather a full picture of the instabilities because like all turbulent models, some of the characteristics are intrinsically 3D phenomena and all experiments are in three dimensions [60]. However, for simplicity the model will be simulated by an array of line vortices.

The annulus injection scheme geometry is simplified as well. The model simulates one and a half fuel and oxidizer injectors in a straight duct. Additionally, the fuel and oxidizer are not differentiated.

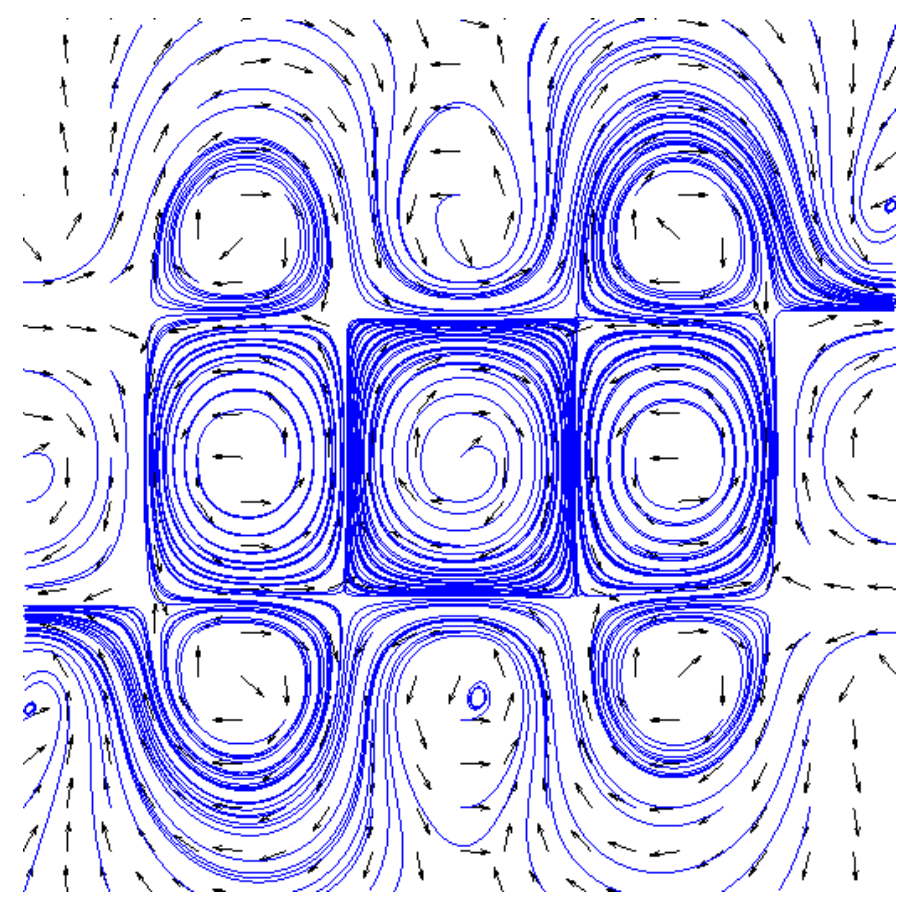

Figure 4.2 Full potential flow simulation of UWRDE mixing. Extraneous vortices were created for boundary conditions to simulate the inner and outer walls. 
The aerodynamics and geometry are considerably simplified. The model consists of an array of 15 vortices (Fig. 4.2). The vortex distances are oriented to closely match the actual location of the walls and injection orifices. The channel width and distance between streams are the same like the UWRDE orifices.

Equation 10 is the general potential function of each line vortex. The potential flow model (Fig. 4.2) was developed from the equation by superimposing an array of line vortices at different positions.

$$
F=-\frac{i \Gamma}{2 \pi} \ln \left(z-z_{0}\right)
$$

$\Gamma$ is the strength of the vortex and $z_{0}$ is the position of the center. The top and bottom vortices are modeled at opposite and half the vortex strength of the center vortices. This was done to simulate the outer and inner walls. Two center edge vortices were used to bound the half injection stream and were at full strength.

Figure 4.3 is the middle section of Fig. 4.2 and is the area that is actually being modeled. The vertical velocity vectors are the injection streams while the horizontal vectors represent the outer and inner walls of the annulus. The resulting flow visualization is similar to flow visualization depicted in Fig. 4.1. It is easy to visualize how mixing is directly related to this flow pattern.

Potential flow makes several important assumptions that affect the validity of the proposed model. The model assumes inviscid, irrotational flow. Due to these assumptions, vorticity can be simulated but flows heavily influenced by vorticity, like the UWRDE vortical mixing scheme, make the model unreliable for deep understanding of the flow characteristics. Regardless this model yields a basic, qualitative understanding of the flow inside the mixing chamber in the UWRDE. 


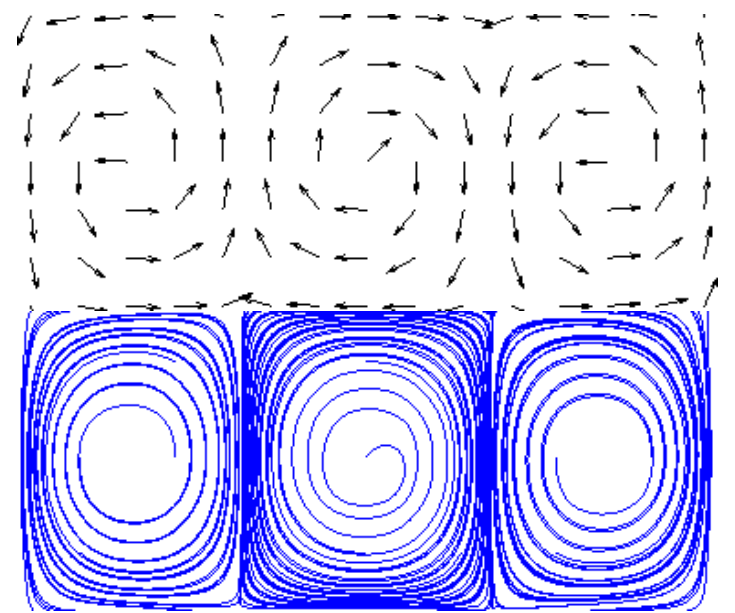

Figure 4.3 Potential flow simulation of UWRDE mixing. The top and bottom image, respectively, show the velocity vector and the streamlines. The actual model from Fig. 4.2 only simulates one and a half jets of fuel and oxidizer.

\subsection{COMPUTATIONAL Simulation}

A higher-level computation tool can give more insight on the internal workings of the UWRDE injection scheme. The following CFD model shows flow visualization with much fewer assumptions than potential flow theory. Figure 4.4 shows a CFD model of the actual UWRDE injection scheme on a 2D plane. The RT instability between fuel and oxidizer is still apparent even though this model assumes the two fluids are the same.

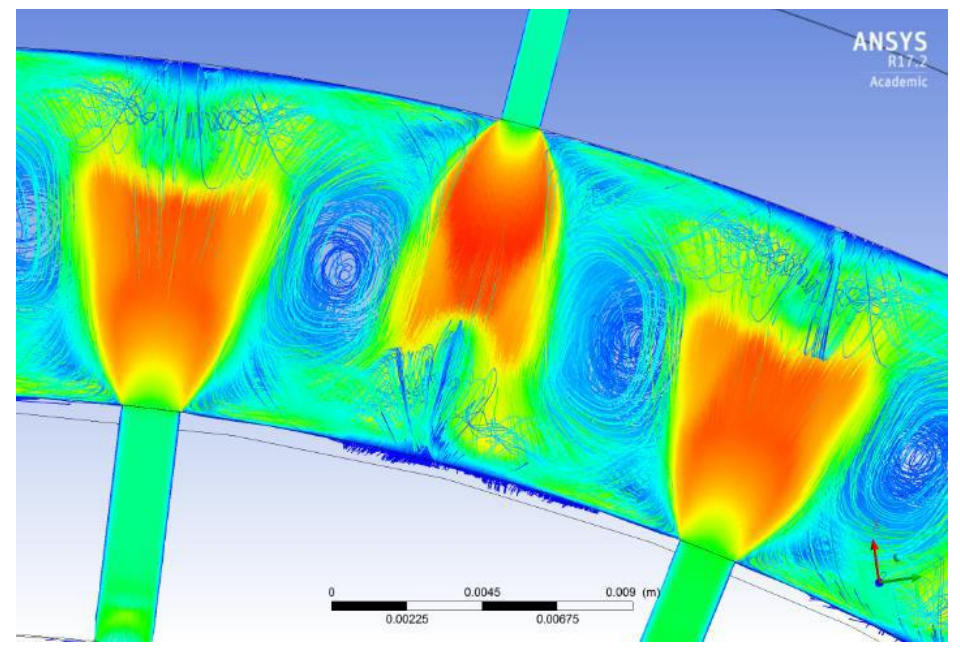

Figure 4.4 CFD model of UWRDE mixing. The 3D model shows velocity streamlines on a 2D plane [44]. 
Figure 4.4 shows how other instabilities would arise like the Kelvin-Helmholtz instability; the flow between the injection orifices are not perfectly circular. This model can be applied to specific fuel and oxidizers or other geometries to yield more insight on the effects of RT instability and mixing quality. Still, the initial pressure difference between the fluid reservoirs provide a density gradient between the fluids. Regardless, this general model still shows how mixing is taking place through the turbulent and high-vorticity field shown.

The potential flow and CFD models reflect similar flow fields. However, the CFD model shows magnitude and can be used to easily inspect other flow properties like pressure, density, and temperature at specific points. Although the two models yield slightly different results, information from both are used to help to better understand the problem.

\subsection{RADIAL INJECTION}

The RT instability in radial injection gives qualitative insight on the effectiveness of the mixing scheme. The more turbulent the mixing, the more mixing will increase. It is expected the initial mixing geometry in the system plays a role in where the detonation zone occurs. This is the focus of the RDE research in the remainder of the paper. The knowledge of the RT instability and various flow models give a better understanding of the mixing in the UWRDE. 


\section{Chapter 5. RESULTS AND DISCUSSION}

The primary goal of CFD simulations was to determine how various geometric and operating parameters influence the length of the mixing zone of radially injected gaseous components. They were carried out with non-reacting flow in a 3-D domain with the inclusion of viscous effects. This approach is sufficient to quantify the degree of mixture variation at all points within the computational domain for comparison with the experimental results for the location of the leading edge of the detonation zone. The cases modeled in this phase of the study are shown in Table 3.4.

The first three injection schemes analyze the effects of increasing mass flux on detonation zone by sweep the mass flux from $10 \mathrm{~kg} / \mathrm{s} / \mathrm{m}^{2}$ to $30 \mathrm{~kg} / \mathrm{s} / \mathrm{m}^{2}$. The fourth scheme was used to bridge the gap between the 154- and 76-mm engine by maintaining the injection scheme and mass flux. The fifth case increased mass flux by almost an order of magnitude in the 76-mm-RDE. The sixth case examined the differences between staggered and impinging injector orifices in the 76-mmRDE at the high mass flux conditions used for the prior case. The results of these comparisons are presented after the details of the CFD set up and validation are discussed here.

\subsection{Detonation Wave Analysis}

The UWRDE is currently not setup for visual imaging. Therefore, to identify certain features of the detonation, high frequency pressure transducers are analyzed through a spectrogram and Fourier transformation. A detailed description of this analysis is shown in past UWRDE papers [46]. 


\subsection{EXPERIMENTAL PROCEDURE AND RESULTS}

Experiments were carried out in the 154-mm-RDE to determine the location of the leading edge of the detonation zone in stoichiometric methane/oxygen propellant by inserting a thermocouple at the center plane of the annulus at various distances (by increments of $\sim 13 \mathrm{~mm}$ ) from the front endwall. Even though the time response of these probes was too slow for measuring arrival time of individual waves, it was sufficient to estimate the location of the leading edge of the detonation zone to within the last recessed thermocouple and the last measurement location. This same technique will be utilized in the new 76-mm-RDE, in conjunction with significantly more instrumentation, to better quantify the location of the detonation zone as the operating parameters are varied.

Figure 5.1 shows the variation of peak temperature along the annular combustor centerline as the axial position of the thermocouple is increased from the front endwall of the 154-mm-RDE. The open plotting symbols indicate the last temperature recorded before the exposed thermocouple bead became unresponsive, which corresponded to the time when it was destroyed by excessive temperatures. The leading-edge location of the detonation zone was then determined by measuring the recessed length of the probe to find the distance from the beginning of the combustion chamber. The distance from the front endwall determined in this manner, normalized by the annular gap dimension is plotted on the right in Fig. 5.1. These preliminary data indicate that the detonation zone leading edge moves closer to the front endwall as mass flux is increased. Correlating the thermocouple data with additional instrumentation is planned when the instrumentation density upgrade has been completed. 

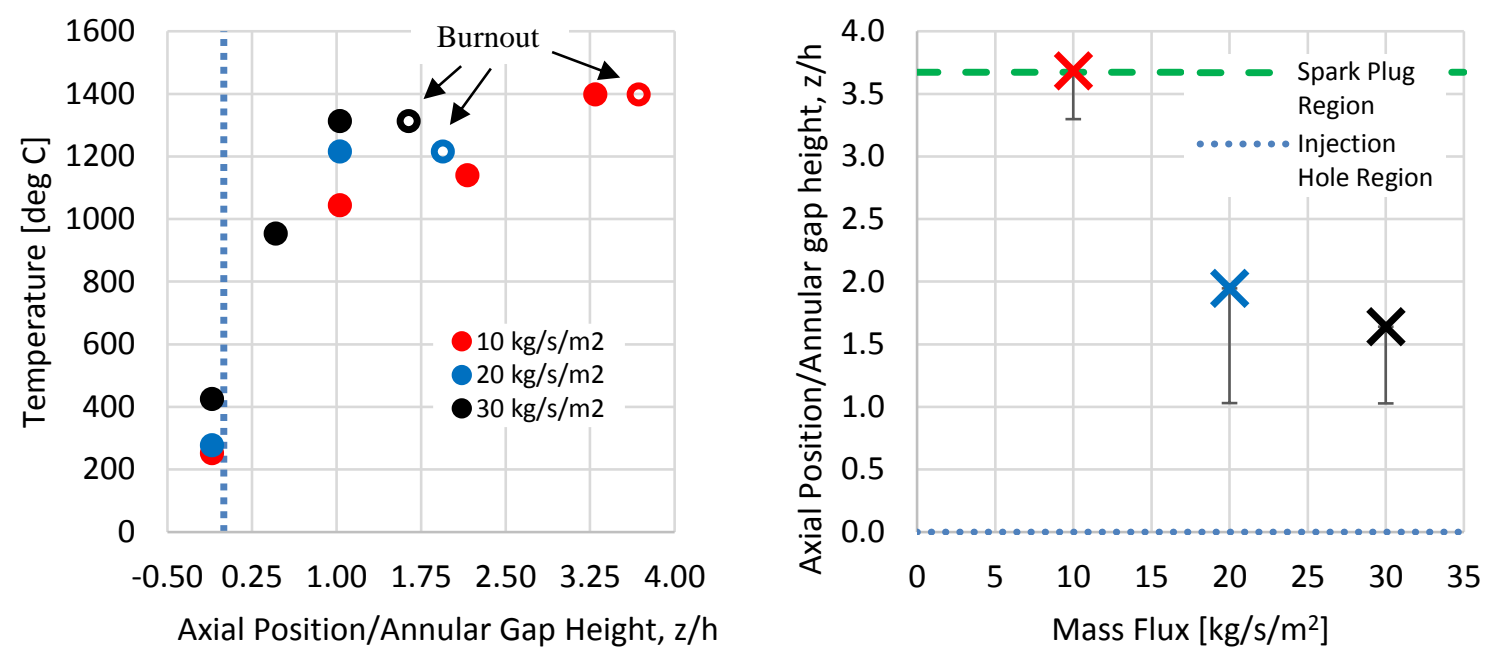

Figure 5.1 Peak temperatures (left) and location of thermocouple inserted through the front endwall of combustor (right).

\subsection{MESH REFINEMENT}

All computational simulations were conducted on a minimum wedge size with symmetric boundary conditions. The 76-mm and 154-mm models were analyzed on a ten- and fifteendegree wedge. This wedge is the minimum size to maximize computational efficiency. The mesh features utilized wall inflation and a maximum of 20 wall boundary layers. The pressure inlet conditions were defined on one side of the front end of the plenum to meet stoichiometric conditions. The outlet boundary was set as an outlet vent at $10 \mathrm{kPa}$ backpressure. The walls were defined as "no slip" wall surfaces. The mesh refinement process focused on changing the element sizes to change the overall number of elements.

The percent difference was taken from the coarsest mesh, which was composed of about $80 \mathrm{~K}$ elements. Since the increase in elements was not precise this method was taken to remove this factor. The goal was to increase the total number of elements by $50 \mathrm{~K}$ for each mesh iteration. The selected mesh was chosen based on decay of percent difference change. The 
coarsest mesh where this qualitative point occurred was selected as the optimal mesh. This process was applied to injection scheme 2-1-4 and the results were applied and scaled by total volume to all the other injection schemes. Figure 5.2 shows the standard deviation from stoichiometric at four different annular gap heights downstream of the injection orifice plane from the coarsest mesh as a function of element number.

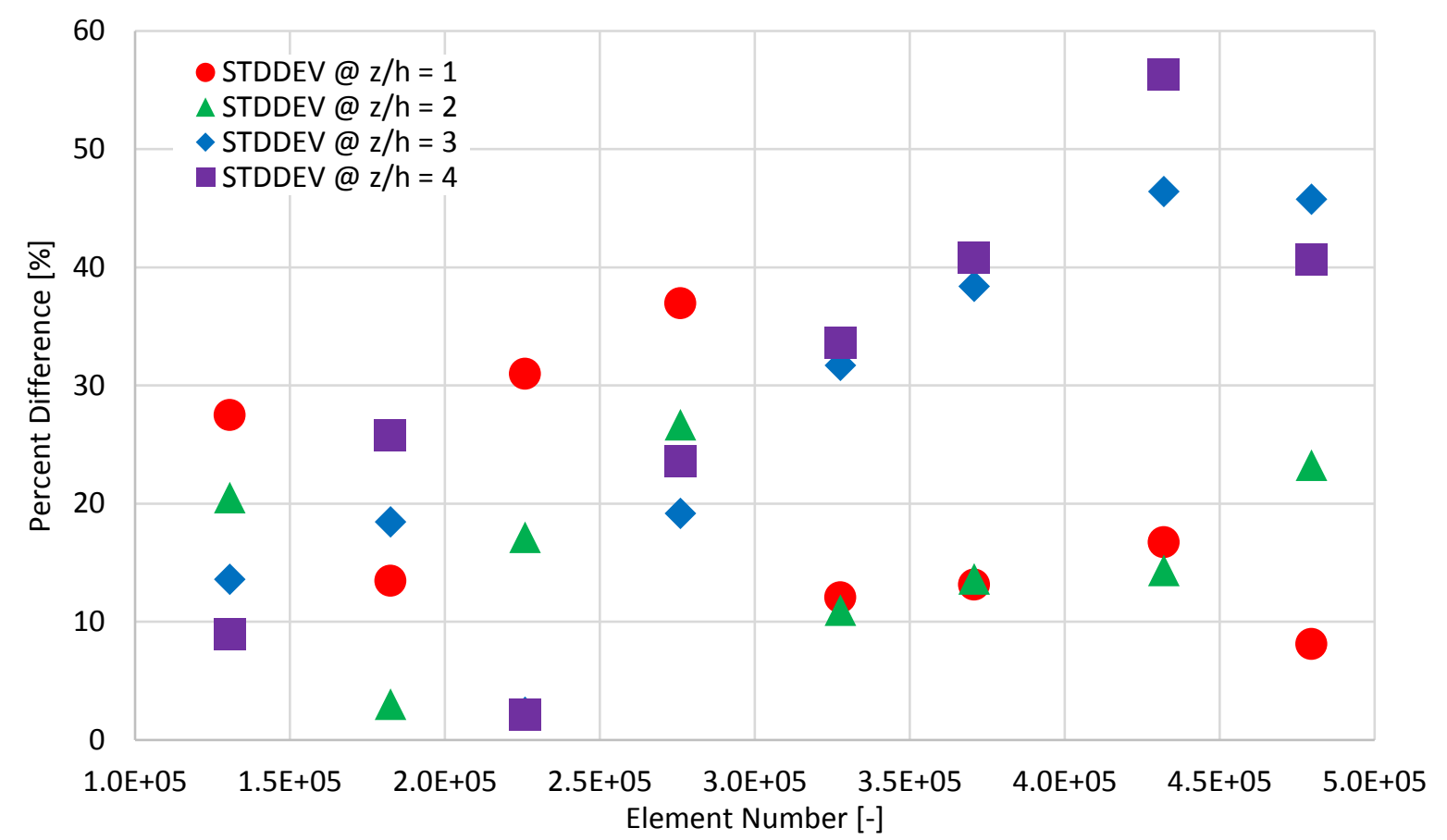

Fig. 5.2 Mesh refinement study summary conducted to select optimal mesh for performing all computation simulations.

There is a fairly reasonable point where the percent differences begin to level and the large fluctuations end. The experimental data from Fig. 5.1 shows the detonation zone occurred between annular gap heights of one and two. The closer gap heights carry more importance for the evaluation of the tool. The optimal mesh selected contained about $330 \mathrm{~K}$ elements. An additional refinement study was conducted on the residual convergence criteria. The selected residual for convergence was acceptable. There is no universal metric for determining convergence. According to the Fluent User's Guide, convergence should be determined based on the behavior, not the actual 
residual values themselves. Figure 5.3 shows the final three meshes used for all remaining simulations.

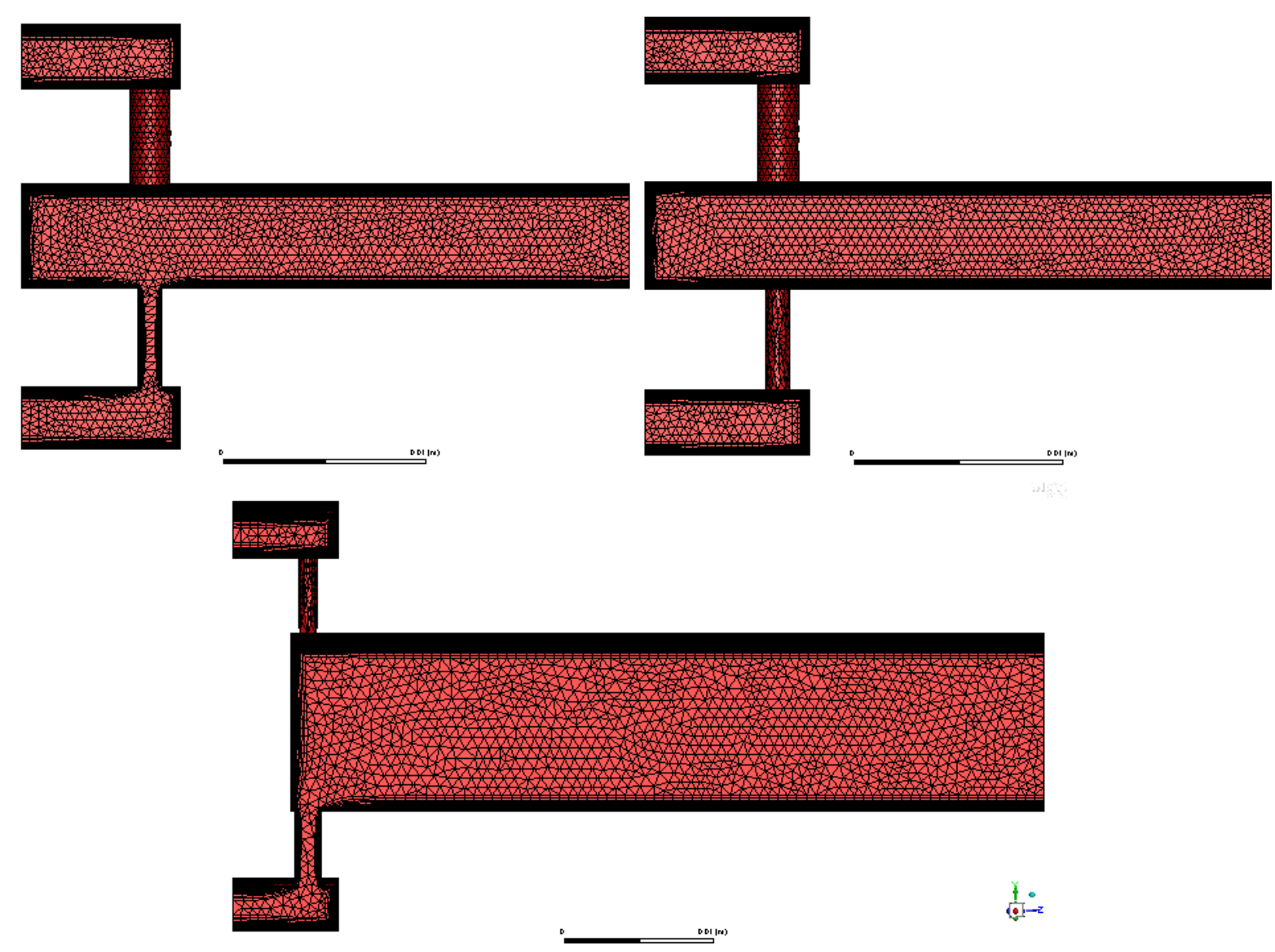

Figure 5.3 Meshes used for all geometries. Injection schemes 2-1-3 and 2-1-4 used the left mesh. Injection scheme 2-2-4 was used the right mesh. Injection schemes 1-1-1, 1-1-2, and 1-1-3 used the bottom mesh.

The difference in geometry between the 154-mm-RDE and 76-mm-RDE is apparent in Fig. 5.3. Most notably, the position of the injection plane in relation to the front endwall for the 154-mmRDE is significantly closer than the 76-mm-RDE. The black are the edges of the mesh and the red are the faces of the injectors and one side of the symmetry plane. The length scale on the images in Fig. 5.3 is $0.01 \mathrm{~m}$. 


\subsection{COMPARISON OF RDE SIZE SCALES}

The 154-mm-RDE and 76-mm-RDE were compared by flowing an equivalent flow of reactants at $30 \mathrm{~kg} / \mathrm{s} / \mathrm{m}^{2}$. If the simulation results for the $154-\mathrm{mm}-\mathrm{RDE}$ can validate the experimental results and the detonation zone occurred at the same nondimensionalized location, the understanding of the detonation zone location could be applied to any scaled version of the RDE in with the same characteristics. In other words, injector schemes 1-1-3 and 2-1-3 results should correlate. There were no correlations for several reasons. The injection orifices were not scaled and did not follow the same spacing. The injection orifices for the 154-mm-RDE were designed to be spaced at the same distance as the annular gap height because this geometry is theoretically the most efficient mixing geometry. However, the 76-mm-RDE was designed to for pressure gain. Theoretical pressure gain required a larger injection area so more orifices were added. The simulations showed the pressure loss with respect to the front endwall at $30 \mathrm{~kg} / \mathrm{s} / \mathrm{m}^{2}$ for the 154 mm-RDE was about 99 percent, while the 76-mm-RDE only had an 81 percent pressure loss. Actual pressure loss of the injectors for the 154-mm-RDE was about 62 percent, while the 76$\mathrm{mm}$-RDE had about 58 percent pressure loss. Initially, the mass flux for the 76-mm-RDE was believed be low enough to result in unchoked orifices but the Mach contours showed this was not the case. Figure 5.4 shows bounded Mach contours and the $76-\mathrm{mm}-\mathrm{RDE}$ at $30 \mathrm{~kg} / \mathrm{s} / \mathrm{m}^{2}$ orifices were choked and was not a problem.

The $270 \mathrm{~kg} / \mathrm{s} / \mathrm{m}^{2}$ was shown for reference to show how the Mach contours should appear for choked orifice operation. The two cases look almost identical and the injection plane, as expected. The cases flow is in excess of Mach two, but were bounded from Mach zero to one to highlight the orifice choking at Mach one. 


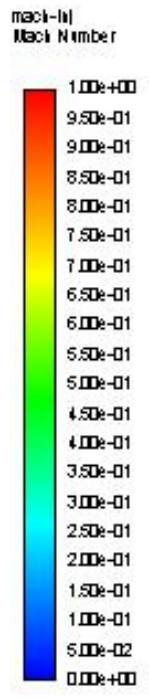

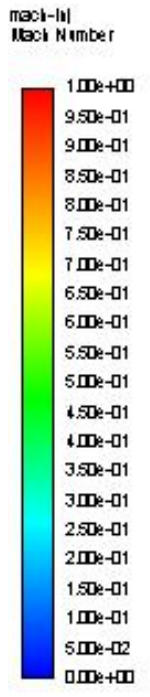

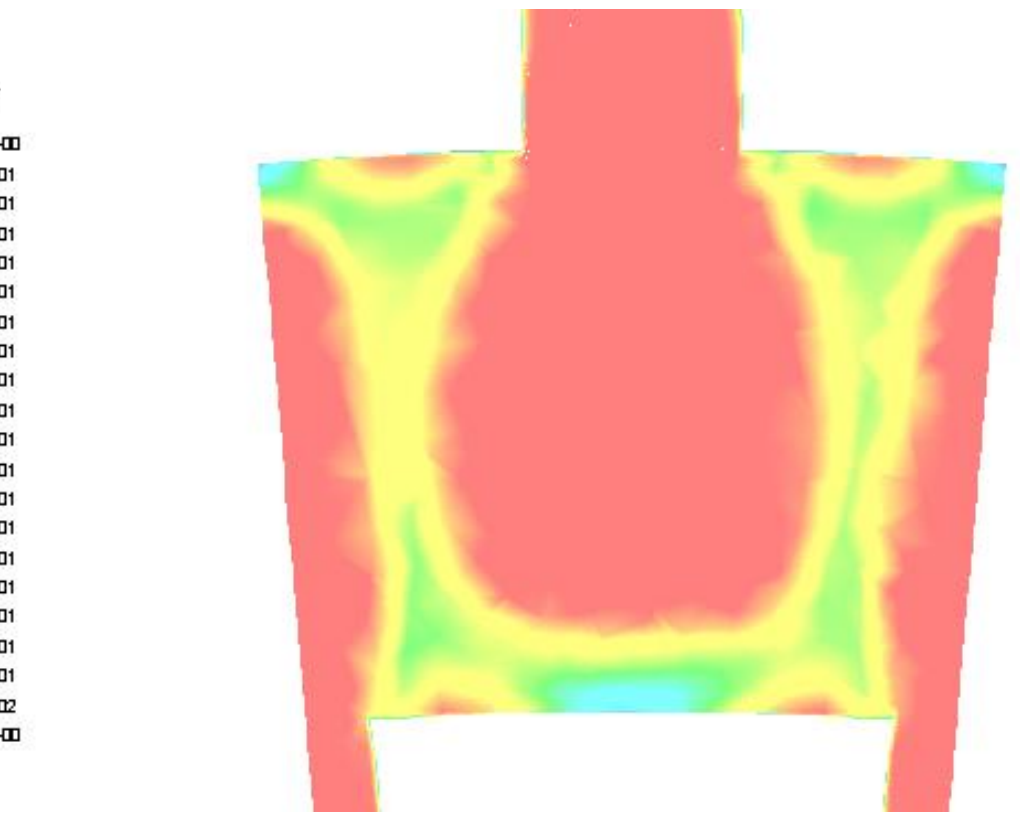

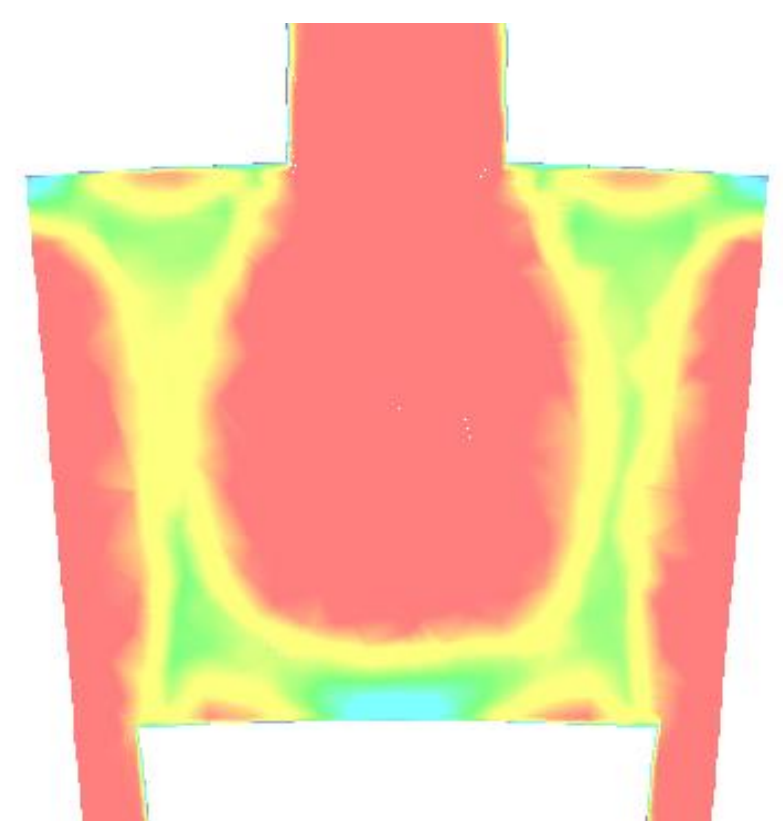

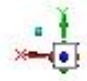

Figure $5.476-\mathrm{mm}-\mathrm{RDE}$ Mach contours at $30 \mathrm{~kg} / \mathrm{s} / \mathrm{m}^{2}$ (top) and $270 \mathrm{~kg} / \mathrm{s} / \mathrm{m}^{2}$ (bottom).

The determination of the pressure loss was another tool that arose from the non-reactive CFD model. Figure 5.5 shows the front endwall pressure contours used to calculate the pressure loss stated previously. As a conservative estimation, the smallest value on the front endwall was used and compared to the total pressure average between the fuel and oxidizer plenum. The fuel plenum pressures were approximately $135 \mathrm{kPa}$ and $1010 \mathrm{kPa}$, respectively for the 76-mm-RDE 
and 154-mm-RDE. The oxidizer plenum pressures were approximately $135 \mathrm{kPa}$ and $1260 \mathrm{kPa}$, respectively for the 76-mm-RDE and 154-mm-RDE. The bounds in the image are adjusted as narrow as possible to show the variation in pressure without losing any information. The knowledge of the general pressure variation on the front endwall needs to be kept in mind. There are limitations from the single point collected from experimental values.

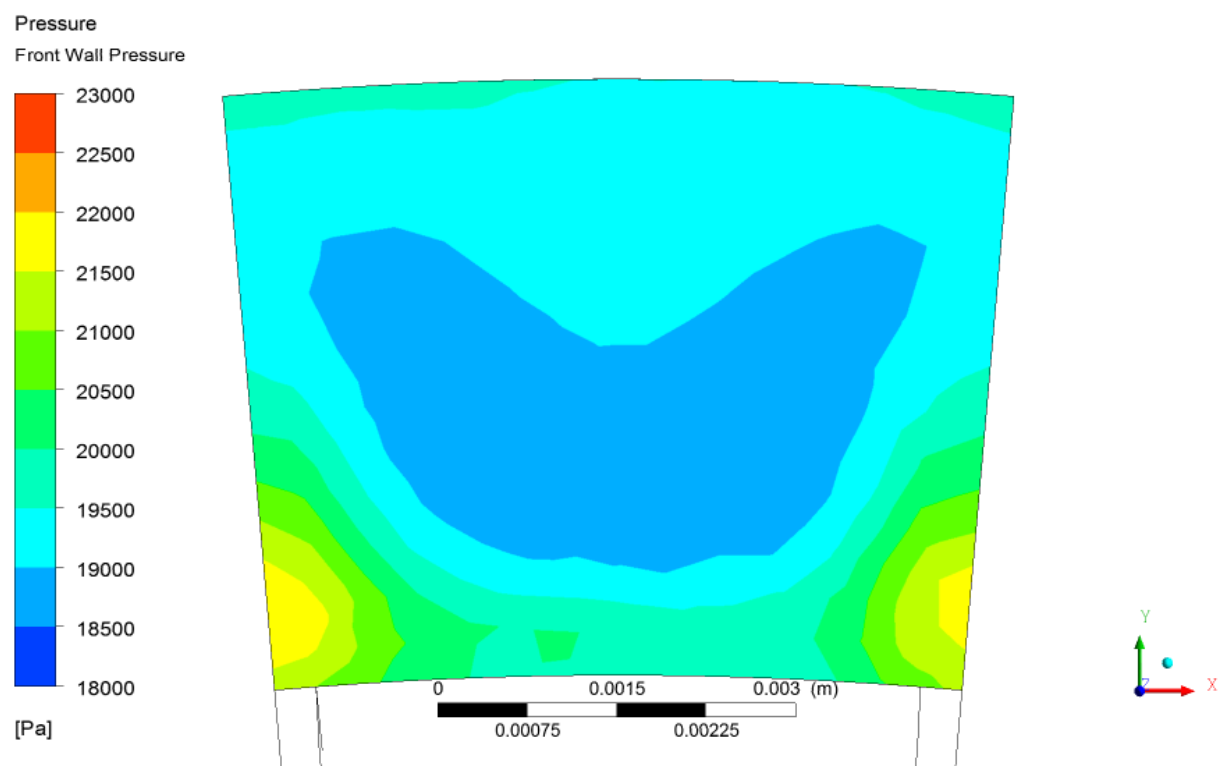

Pressure

Pressure Front Wall

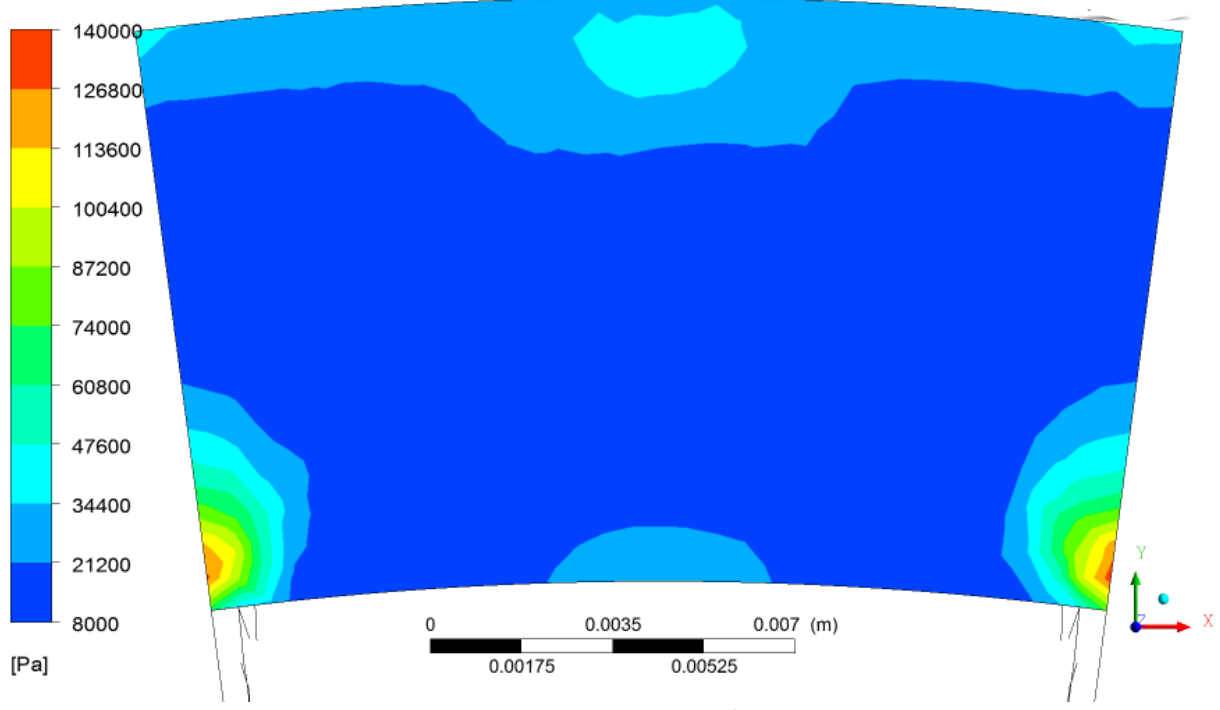

Figure 5.5 Pressure contours on front endwall at $30 \mathrm{~kg} / \mathrm{s} / \mathrm{m}^{2}$ for the $76-\mathrm{mm}-\mathrm{RDE}$ (top) and 154-mm-RDE (bottom). 
The model allows the user to track the change in total and static pressure. Figure 5.6 shows the pressure ratio averaged at various axial position for the size scale comparison. The data summarized in Fig. 5.6 is extracted from the simulation results shown in Figs. B.1 and B.2, which are tabulated in Table B.1 and B.2. Note, the axial positions averaged before and at the injection plane include the fuel and oxidizer plenums. This plot shows total pressure is greater than the static pressure throughout. After the injection orifices, the pressure ratio rises sharply and slowly decreases. This occurs due to the drop in static pressure as the flow accelerates downstream and the total pressure decreases from entropy losses. The overall pressure discussion is not as relevant to the thesis objectives but it was important enough to note for the purposes of completeness and to highlight another tool from the non-reactive simulations.

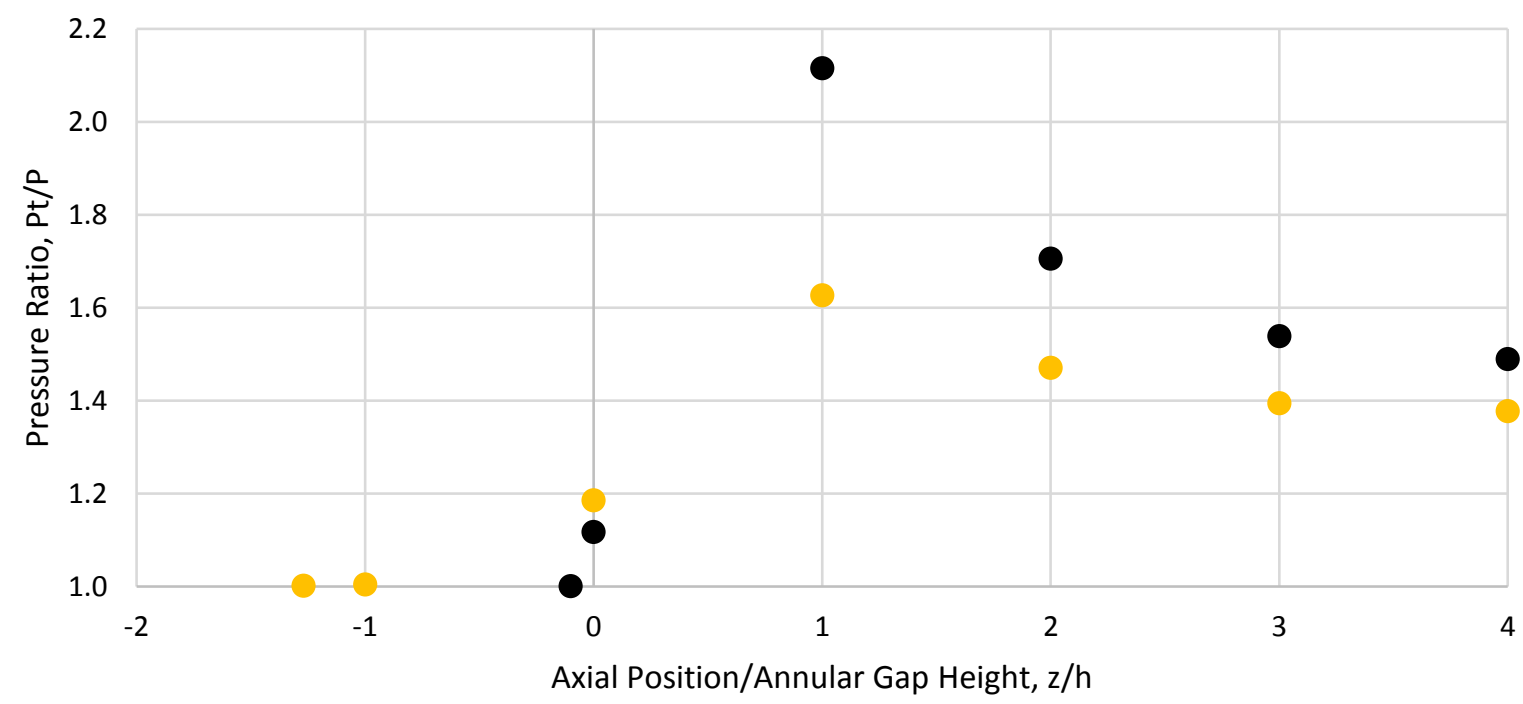

Figure 5.6 Pressure ratio at $30 \mathrm{~kg} / \mathrm{s} / \mathrm{m}^{2}$ for the 76-mm-RDE (yellow) and 154-mm-RDE (black). Pressure contours shown in Figs. B.1 (yellow) and B.2 (black).

\subsection{INJECTION SCHEME Velocity PROFILE}

The injection velocity profile becomes less important when quantifying the quality of mixing by Turbulence Kinetic Energy (TKE) and vorticity. However, the injection velocity profile does give 
a nice visualization and understanding of how the two species first interact with each other. By analyzing the injection velocity profile, it is clear staggered/impinged geometries and engine size mix differently.

The staggered or transverse and impinged profiles in Fig. 5.7 shows the peak velocity from the fuel orifice greater than the speed of sound. By inspection of the flow field around the orifice exit, the flow expands and the slower particles act as a divergent nozzle. This expansion allows the flow move past the speed of sound. The approximate speed of sound for methane and oxygen at $300 \mathrm{~K}$ is about $450 \mathrm{~m} / \mathrm{s}$ and $330 \mathrm{~m} / \mathrm{s}$, respectively. The momentum ratio (methane to oxygen) of injector range anywhere from about 0.21 to 0.24 for any injection scheme and mass flux simulated.
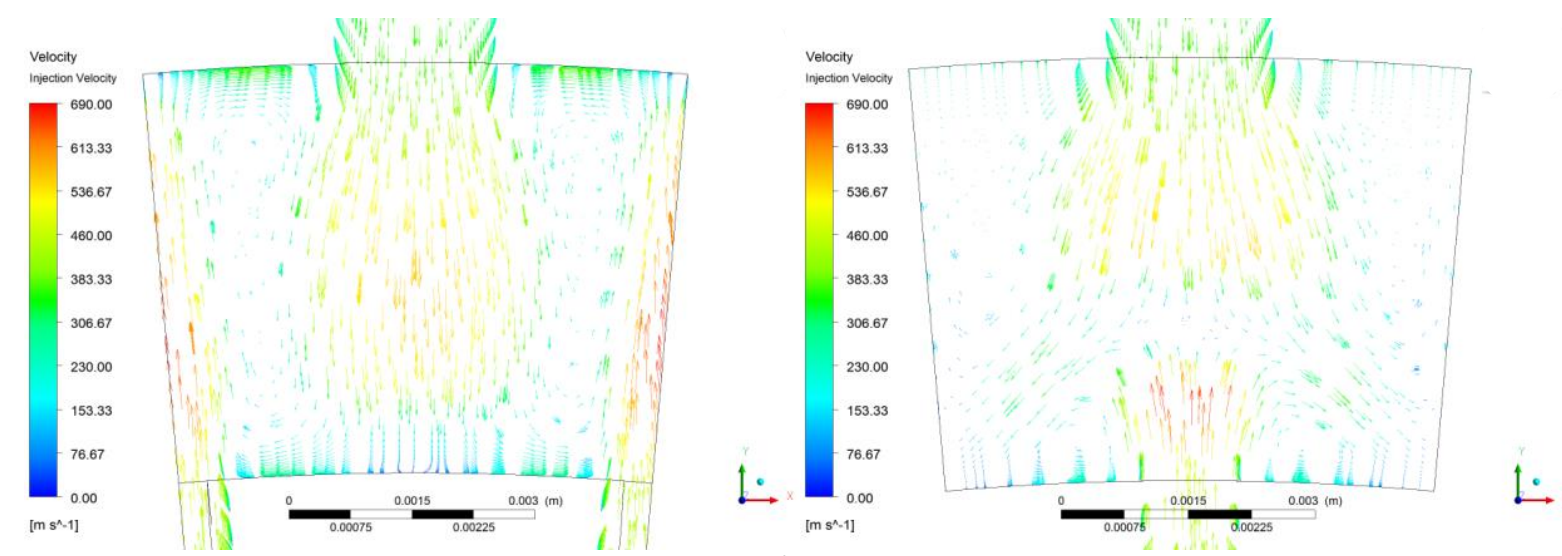

Figure 5.7 Velocity profiles for the $270 \mathrm{~kg} / \mathrm{s} / \mathrm{m}^{2}$ cases. Injection scheme 2-1-4 (left) and 2-2-4 (right).

By overlaying the velocity profile with the TKE contours, it shows are the center of the swirling motion produces the most TKE. The larger the TKE magnitude indicates a higher quality of mixing. Increased mixing results in a shorter distance to reach stoichiometric conditions. Figure 5.8 shows TKE contours overlapped by the same velocity profiles as Fig. 5.7. 

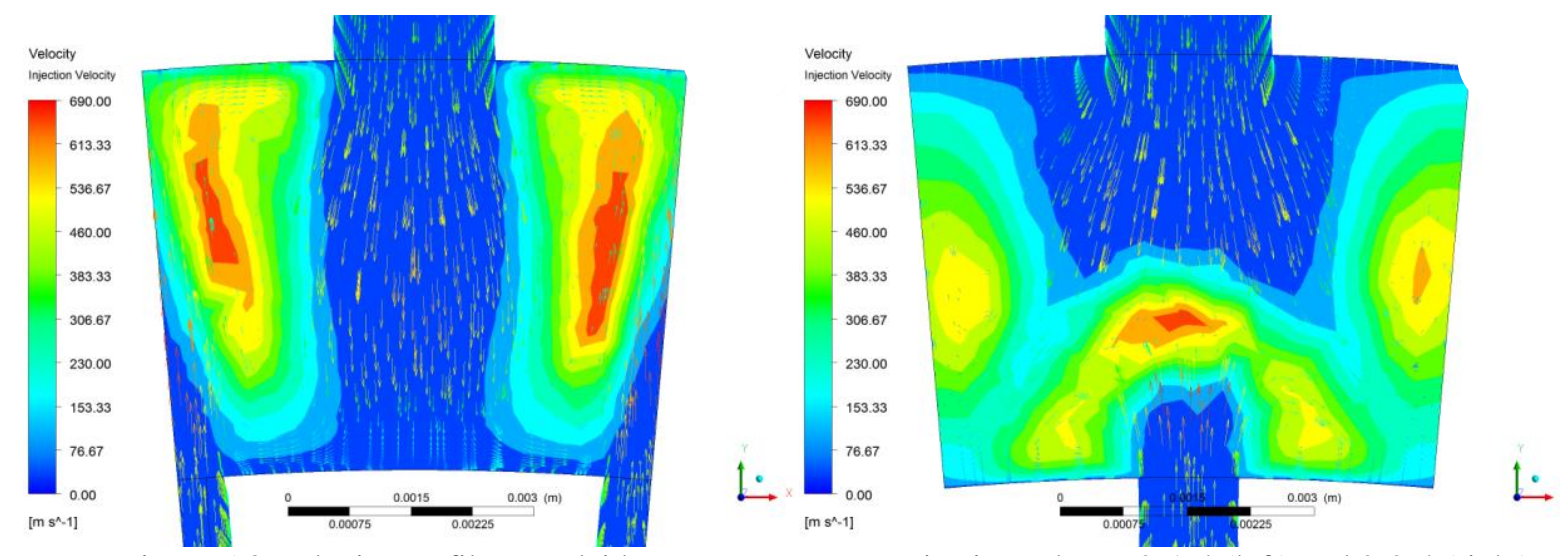

Figure 5.8 Velocity profiles overlaid on TKE contours. Injection scheme 2-1-4 (left) and 2-2-4 (right).

\subsection{MASS FLUX CONSIDERATIONS}

The analysis of the velocity profiles should show no change in velocity magnitude at this plane. This makes sense and is expected because the flow is choked while exiting the injection orifices. Figure 5.9 shows little to no distinction between the two profiles. This occurs because both orifices are choked as shown in Fig. 5.4.
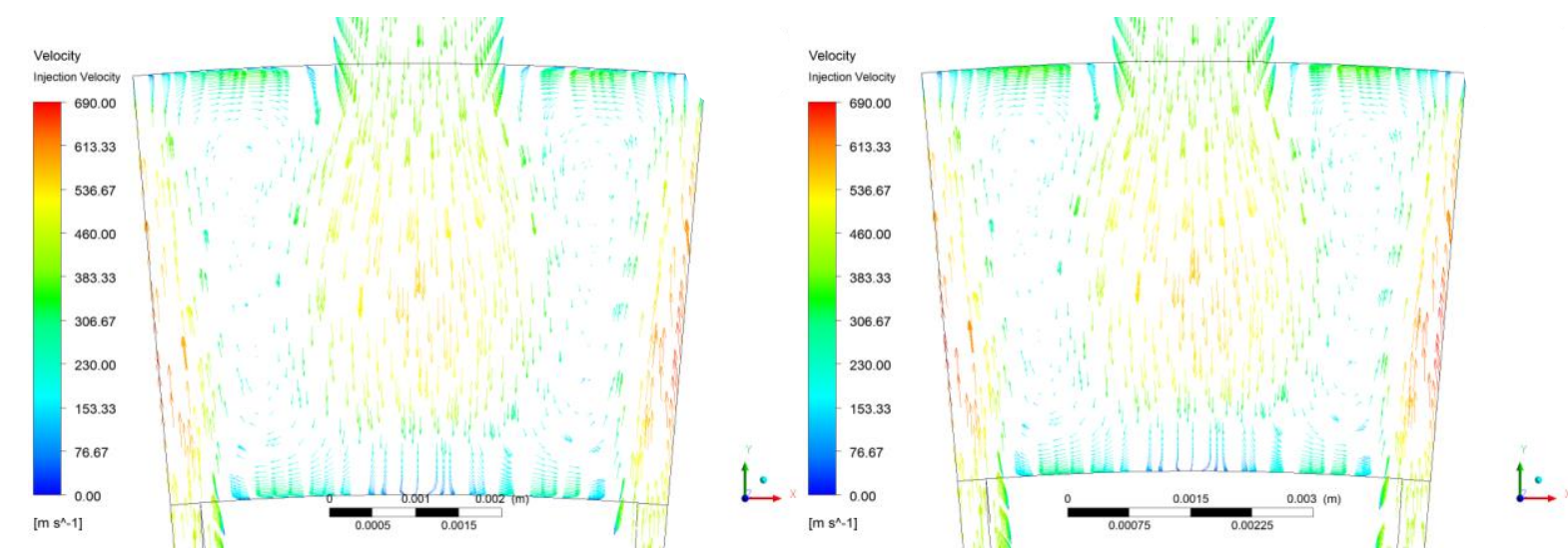

Figure 5.9 Velocity profiles of different mass flux $30 \mathrm{~kg} / \mathrm{s} / \mathrm{m}^{2}$ (left) and $270 \mathrm{~kg} / \mathrm{s} / \mathrm{m}^{2}$ (right) cases. Injection scheme 2-1-3 (left) and 2-1-4 (right). See Fig. B.2 for remaining injection scheme contours.

Although shown in experimentation higher mass flows increase mixing, Fig. 5.10 shows an unexpected result, mixing decreases with an increase in mass flux. The equivalence ratio for injection scheme 1-1-3 approaches unity further downstream when compared to 1-1-1 or 1-1-2. 


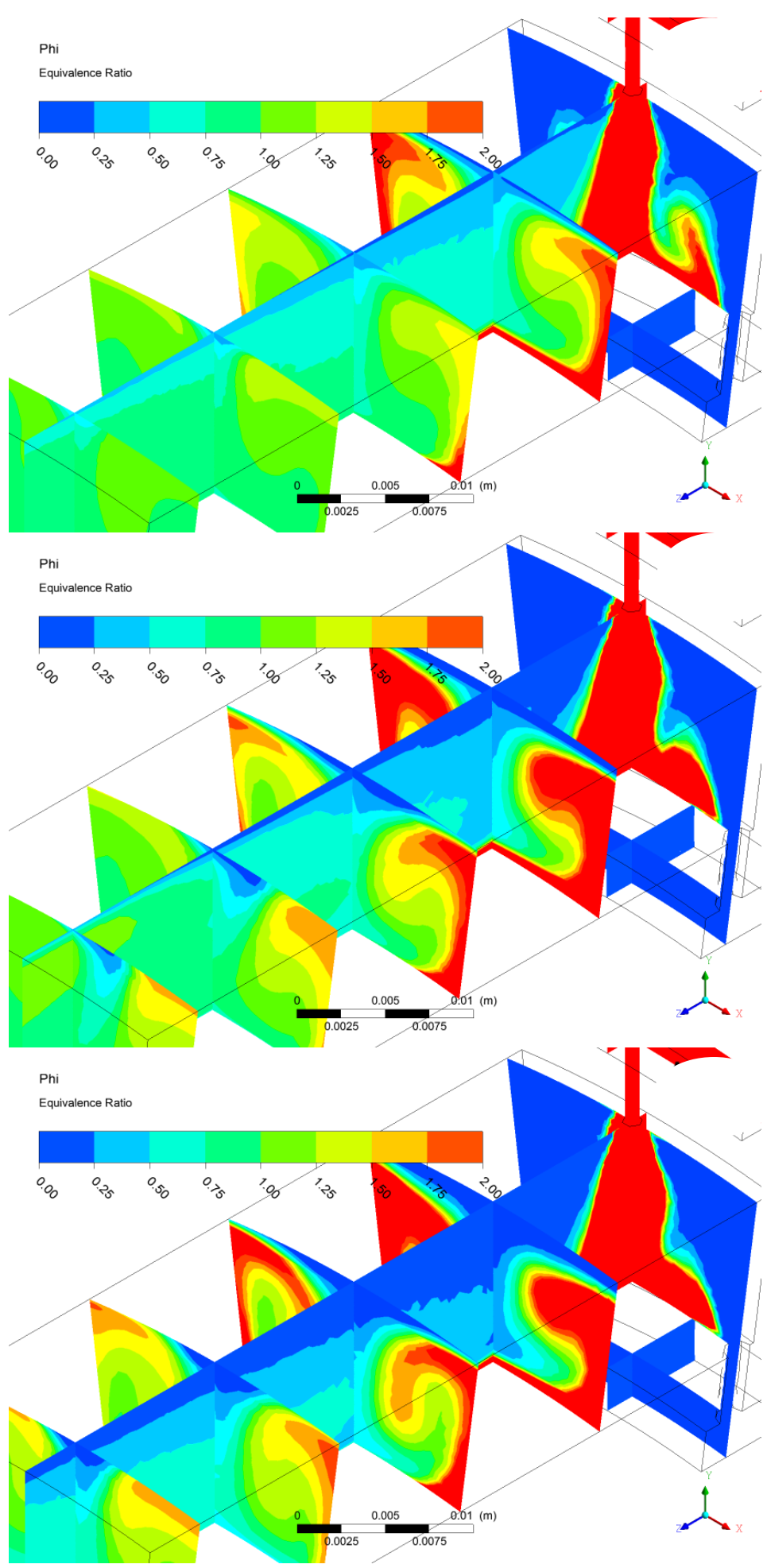

Figure 5.10 Equivalence ratio contours for 154-mm-RDE at varying mass flux. $10 \mathrm{~kg} / \mathrm{s} / \mathrm{m}^{2}$ (top), $20 \mathrm{~kg} / \mathrm{s} / \mathrm{m}^{2}$ (middle), $30 \mathrm{~kg} / \mathrm{s} / \mathrm{m}^{2}$ (bottom). 


\subsection{COMPARISON WITH EXPERIMENT}

The average equivalence ratio across the planes downstream were analyzed and compared to the actual data from the thermocouple experiments in the 154-mm-RDE. Ideally, the detonable equivalence ratio closest to the front endwall will match or correlate with the location the thermocouple failed or the detonation zone was experimentally determined. Figure 5.11 summarizes the simulation results.

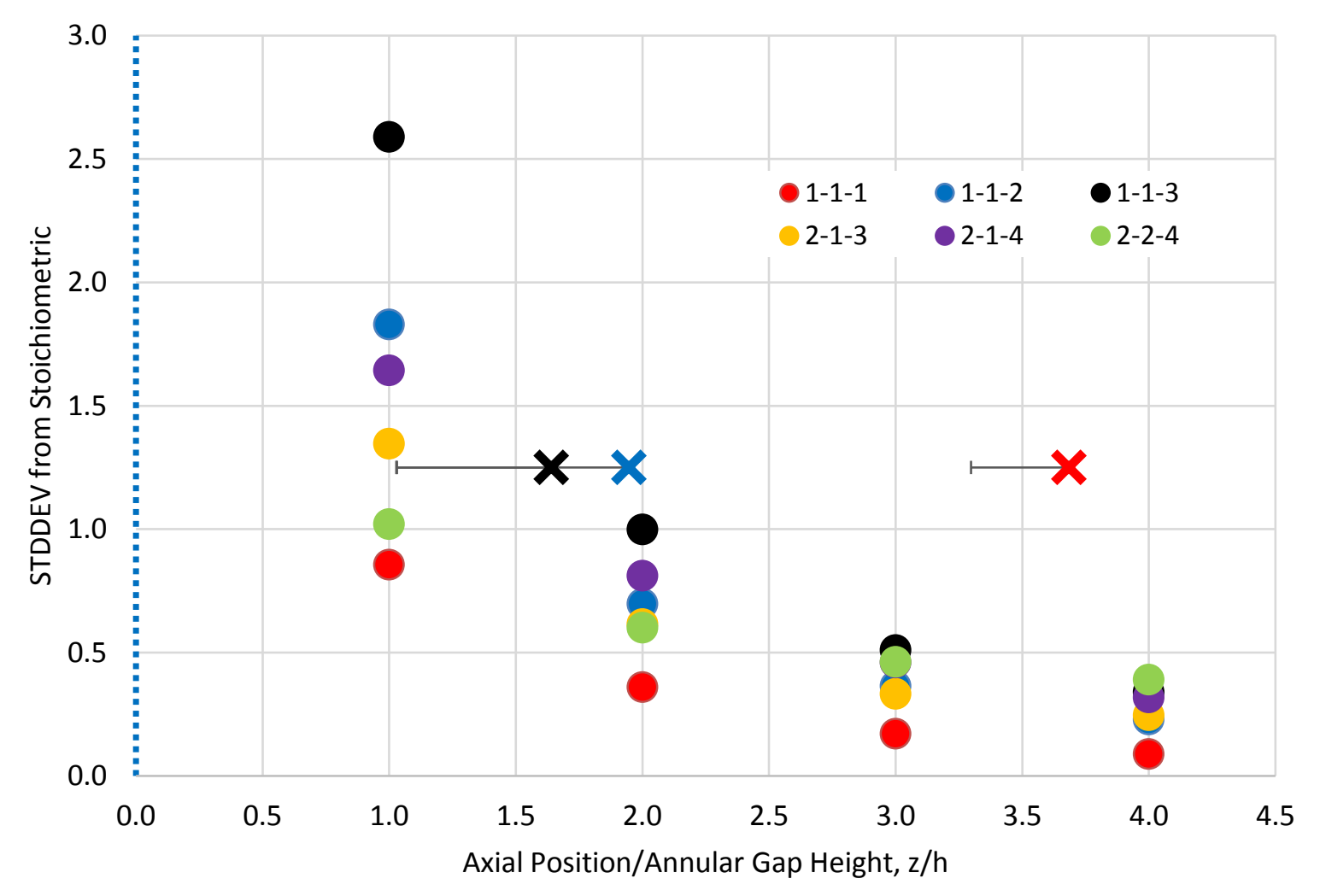

Figure 5.11 Graphical summary of simulation results.

The first point (closest to left) within the bounds of the lean and rich limits for the 76-mm and 154-mm-RDE shows is the prediction to where the detonation zone will stabilize. The rich and lean flammability limit equivalence ratios were determined from the ternary diagram in Fig. 1.5. These limits are the most restrictive limits; the 76-mm and 154-mm-RDE will have smaller 
limits. Additionally, the experimental results (Fig. 5.11) are included as "x" symbols to denote where the detonation zone is located and were placed at an arbitrary standard deviation from stoichiometric of 1.25 . The standard deviation from unity is the best was to describe the level of mixedness. A standard deviation to show completed mixing is zero but does not occur in the first four annular gap heights downstream of the injection orifice. The correlation between simulation results and experimental data point show the detonation zone is stabilize between 0.5 and 1.5 standard deviations.

Figure 5.12 shows a graphical representation of how the equivalence ratio varies with axial position. Each plane normal to the z-direction was averaged and plotted on Fig. 5.11 for each injection scheme. The variation in equivalence ratio decreases as the axial position increases.

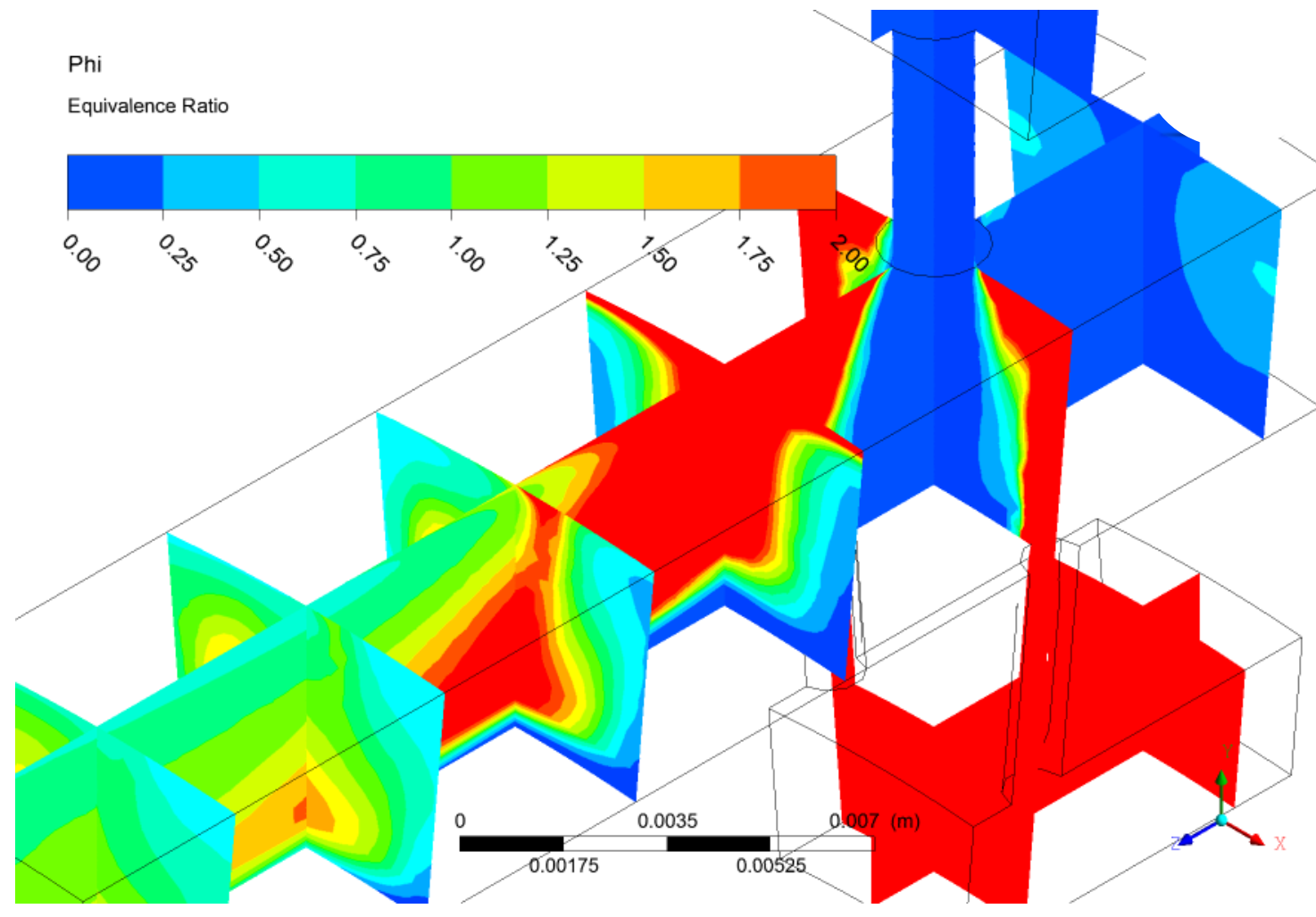

Figure 5.12 Equivalence ratio contour for 76-mm-RDE at $270 \mathrm{~kg} / \mathrm{s} / \mathrm{m}^{2}$ (Injection scheme 2-1-4). See Fig. B.3 for remaining injection scheme contours. 
As the axial position increases the equivalence ratio approaches unity and the variation in the contours becomes nonexistent.

\subsection{MiXing Quality Measures OF Merit}

Two additional properties were considered to quantify the level of mixing — vorticity and TKEin addition to the average equivalence ratio along the axial combustor position. This was analyzed as another method for analyzing the efficiency of mixing. The actual quantity of vorticity or TKE was not considered; the quantity relative to different cases was all that was relevant for the purposes of this paper. Vorticity is useful for measuring the tendency of the fluid to rotate. Vorticity provides a direct relation to mixing as described in the Introduction section. TKE is the mean kinetic energy per unit mass associated with eddies in turbulent flow. Physically, TKE is the RMS of velocity fluctuations or the average turbulence normal stresses. The three-dimensional space was cumbersome to analyze, so the space for measuring mixing quality was simplified to the injection plane. The normalized TKE and vorticity in the injection plane were analyzed to compare to various injection schemes. TKE was normalized to the squared averaged fuel and oxidizer velocity and vorticity was normalized to the annular gap height and averaged fuel and oxidizer velocity. Equations 11-13 show how TKE and vorticity were nondimensionalized.

$$
\begin{gathered}
\bar{u}=\frac{u_{f u e l}+u_{o x}}{2} \\
\widehat{T K E}=\frac{T K E}{\bar{u}^{2}} \\
\widehat{\omega}=\frac{\omega}{\bar{u} / h}
\end{gathered}
$$


Table 5.1 shows the nondimensional TKE and vorticity compared with the equivalence ratio standard deviation from stoichiometric determined from CFD simulations. In terms of spatial mixing, the standard deviation from stoichiometric reaches zero percent in the shortest distance results in the best mixing. The TKE and vorticity were based on magnitude of the peak values injection plane. Upon first inspection there does not appear to be a strong correlation between TKE and vorticity with equivalence ratio standard deviation. The simulations attempted to find a relation between the different size engines and the normalized peak TKE and vorticity, but the results do not show an outstanding relation between mixing effectiveness and TKE/vorticity.

Table 5.1 Comparison of mixing measures of merit.

\begin{tabular}{cccccc}
\hline $\begin{array}{c}\text { Configuration } \\
\text { Designator }\end{array}$ & $\begin{array}{c}\text { STDDEV at } \\
\mathrm{z} / \mathrm{h}=2\end{array}$ & $\begin{array}{c}\text { STDDEV at } \\
\mathrm{z} / \mathrm{h}=3\end{array}$ & $\begin{array}{c}\text { STDDEV at } \\
\mathrm{z} / \mathrm{h}=4\end{array}$ & $\begin{array}{c}\text { Normalized } \\
\text { Vorticity at } \\
\mathrm{z} / \mathrm{h}=0\end{array}$ & $\begin{array}{c}\text { Normalized TKE at } \\
\mathrm{z} / \mathrm{h}=0\end{array}$ \\
\hline $1-1-1$ & 0.36 & 0.17 & 0.09 & $9.04 \mathrm{E}+03$ & $1.16 \mathrm{E}-01$ \\
$1-1-2$ & 0.70 & 0.36 & 0.23 & $1.25 \mathrm{E}+04$ & $8.48 \mathrm{E}-02$ \\
$1-1-3$ & 1.00 & 0.51 & 0.34 & $1.44 \mathrm{E}+04$ & $9.04 \mathrm{E}-02$ \\
$2-1-3$ & 0.62 & 0.33 & 0.25 & $5.22 \mathrm{E}+03$ & $1.40 \mathrm{E}-01$ \\
$2-1-4$ & 0.81 & 0.46 & 0.32 & $1.65 \mathrm{E}+04$ & $1.23 \mathrm{E}-01$ \\
$2-2-4$ & 0.60 & 0.46 & 0.39 & $1.63 \mathrm{E}+04$ & $9.59 \mathrm{E}-02$ \\
\hline
\end{tabular}

In order to determine if there is a direct a relation between the normalized vorticity and rapidity of mixing, the effectiveness of mixing was first ranked by how quickly the standard deviation approaches zero as opposed to the actual simulation values. If that is the case, the normalized vorticity values will correlate with mixing effectiveness. This can be analyzed by comparing the exponential curve fits for the data points at four axial stations. Figure 5.13 shows the plot with best fit curves and the corresponding equations. This is the same plot as Fig. 5.11 with trendlines and equations. Table 5.2 summarizes the exponential decay trendline argument from Table 5.1. 


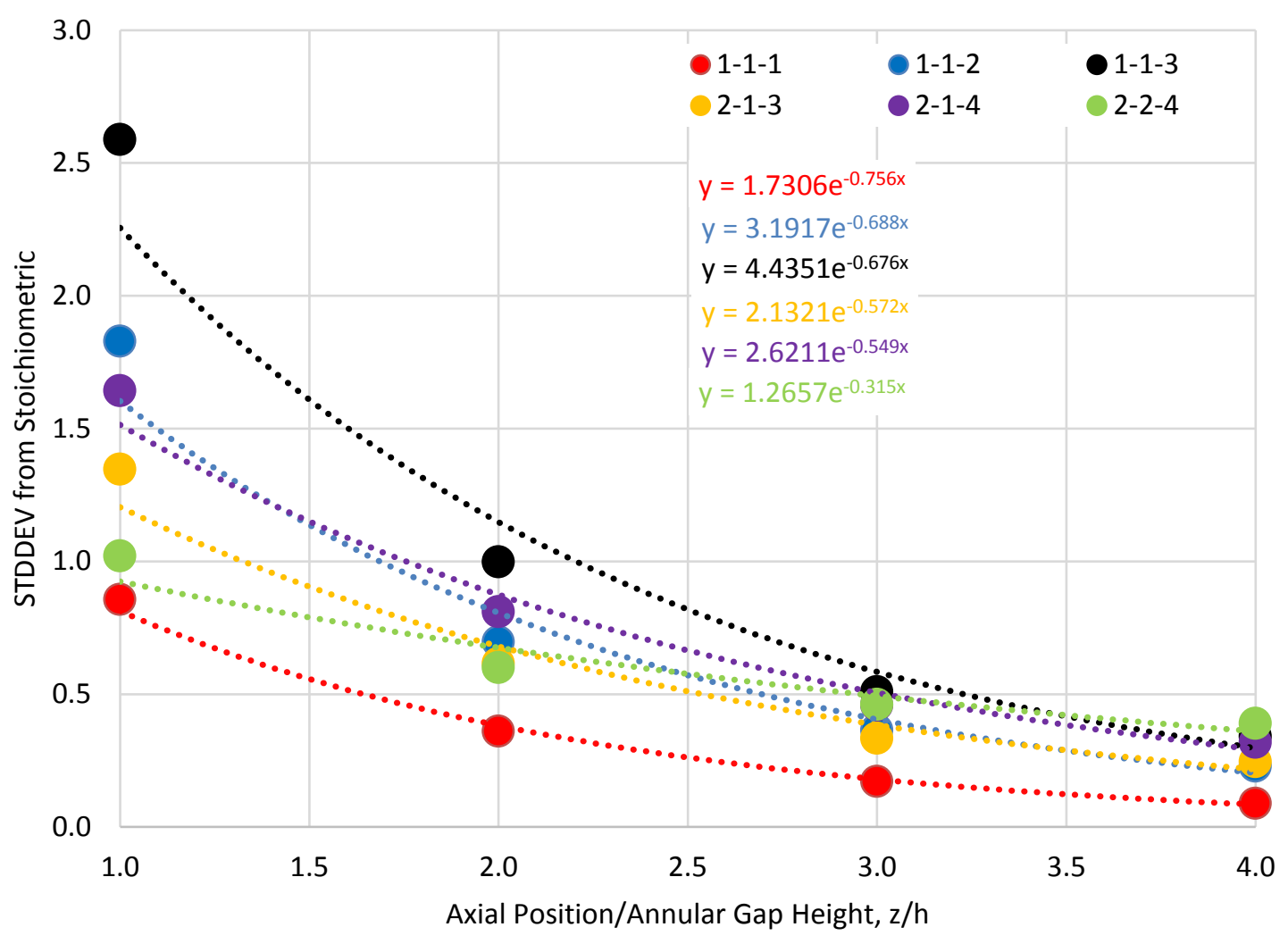

Figure 5.13 Graphical summary of results with exponential trendlines.

The more negative the exponent value in the exponential decay rate, the shorter the distance is required for the mixture to attain near stoichiometric conditions. The absolute value of the exponent in the decay rate curve it was found to decrease with increasing mass flux for both RDE size-scales and injector configurations.

Table 5.2 Mixing measures of merit with exponential decay rate.

\begin{tabular}{c|ccc}
\hline $\begin{array}{c}\text { Configuration } \\
\text { Designator }\end{array}$ & $\begin{array}{c}\text { Trendline } \\
\text { Exponential } \\
\text { Decay Rate }\end{array}$ & $\begin{array}{c}\text { Normalized } \\
\text { Vorticity }\end{array}$ & $\begin{array}{c}\text { Normalized } \\
\text { Turbulence Kinetic } \\
\text { Energy }\end{array}$ \\
\hline $1-1-1$ & 0.756 & $9.04 \mathrm{E}+03$ & $1.16 \mathrm{E}-01$ \\
$1-1-2$ & 0.688 & $1.25 \mathrm{E}+04$ & $8.48 \mathrm{E}-02$ \\
$1-1-3$ & 0.676 & $1.44 \mathrm{E}+04$ & $9.04 \mathrm{E}-02$ \\
$2-1-3$ & 0.572 & $5.22 \mathrm{E}+03$ & $1.40 \mathrm{E}-01$ \\
$2-1-4$ & 0.549 & $1.65 \mathrm{E}+04$ & $1.23 \mathrm{E}-01$ \\
$2-2-4$ & 0.315 & $1.63 \mathrm{E}+04$ & $9.59 \mathrm{E}-02$ \\
\hline
\end{tabular}


The exponential decay rate decreased as mass flux increased for each configuration. The TKE measurement of merit has the same trend, however, the vorticity tended to increase with increasing mass flux. Note that in the 76-mm-RDE at the high mass flux $\left(270 \mathrm{~kg} / \mathrm{s} / \mathrm{m}^{2}\right)$, the impact of injector geometry on both TKE and vorticity was relatively small compared with the changes in decay rate exponent.

In order to visualize normalized TKE and its relation with equivalence ratio standard deviation from stoichiometric, Fig. 5.14 shows how the contours become more homogenous as the flow moves further downstream. Although the injection plane contour was previously shown in Fig. 5.8, this shows how the TKE develops as flow progresses further downstream. As expected, the TKE magnitudes decrease to show the how mixing effectiveness may be described by other measures of merit. The smallest standard deviation or largest decay rate value relates to the highest normalized TKE values when analyzing the two engines independently.

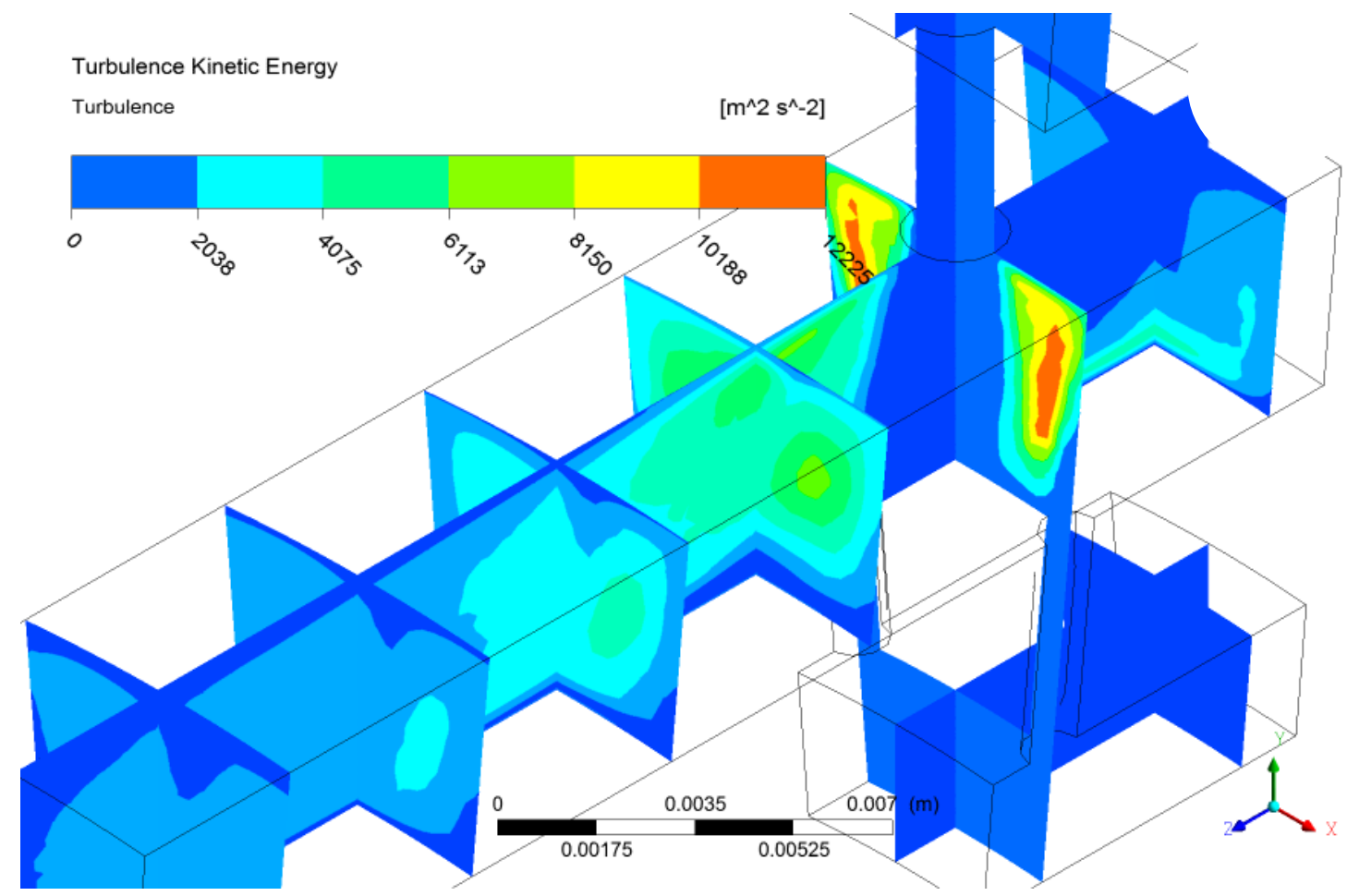

Figure $5.14 \mathrm{TKE}$ contour for 76-mm-RDE at $270 \mathrm{~kg} / \mathrm{s} / \mathrm{m}^{2}$ (Injection scheme 2-1-4). Compare with Fig. 5.11 . 


\section{Chapter 6. CONCLUSION AND RECOMMENDATIONS}

The development of the non-reactive gas model has shown mixing of gaseous methane/oxygen under 0.5 standard deviations within the first four channel heights. The comparison of non-reactive simulation results yields flow characteristics and provides visual and numerical values, otherwise not available through experimentation.

The simulations showed increased mixing with staggered, radial injection geometry over impinged geometry. Mixing efficiency and pressure loss improved with lower mass flux due to mixing occurring in the choked radial direction. The comparison of the simulation results to the experimental results were inconclusive. The summary of the experimental detonation zone location shown below in Table 6.1 are the same results from Fig. 5.1, with the axial location values from the injection plane being nondimensionalized by annular gap height. The reported numbers are the experimental average with the respective uncertainty. The experimental portion of this paper is lacking and needs to be expanded.

Table 6.1 Summary of detonation zone location results.

\begin{tabular}{cccc}
\hline Configuration & Type K Thermocouple & Type C Thermocouple & Tungsten Wire \\
\hline $1-1-1$ & $3.68 \pm 0.39$ & N/A & N/A \\
$1-1-2$ & $1.95 \pm 0.92$ & N/A & N/A \\
$1-1-3$ & $1.64 \pm 0.61$ & N/A & N/A \\
\hline
\end{tabular}

\subsection{RECOMMENDATIONS}

First and foremost, the data collected from the type K thermocouple needs to be repeated multiple times and an uncertainty analysis needs to be performed on the collected data. Experimental results should be collected on the 76-mm-RDE to validate the simulation results.

The detonation zone location instrumentation to be used on the 76-mm-RDE should be applied to 
the 154-mm-RDE. The results should be repeated multiple times with the higher rated type C thermocouple (peak temperature $\sim 2600 \mathrm{~K}$ ) and tungsten wire instead of the single type $\mathrm{K}$ thermocouple (peak temperature $\sim 1350 \mathrm{~K}$ ) in an attempt to get closer to the detonation zone. The tungsten wire should be used more extensively due to financial benefits. Tungsten wire material properties have similar heat resistance values to the type $C$ thermocouple but without the expensive electrical components. Additional data are necessary for statistical correlations to get more accurate, repeatable results. Ideally, a mass flow controller to yield specific, consistent mass flux and equivalence ratios should be installed in the system. To build further from this experiment, it is recommended to analyze thrust measurements on the system. To build further from simulations, investigate transient effects and couple reactive flow and thermal effects.

\subsection{CONCLUSIONS}

The use of a three-dimensional, steady, non-reactive, multiple species gaseous model should be investigated further to use for preliminary RDE design and analysis. The model relevance has been shown and can be used as a parametrical tool. The inclusion of chemical reactions will ultimately be needed to ascertain the most effective mixing schemes. 


\section{BIBLIOGRAPHY}

[1] Lu, F.K., Bruan, E.M., "Rotating Detonation Wave Propulsion: Experimental Challenges, Modeling, and Engine Concepts," Journal of Propulsion and Power, Vol. 30, No. 5, pp. 1125-1142, 2014. DOI: 10.2514/1.17656.

[2] Mattison, D.W., Brophy, C.M., Sanders, S.T., Ma, L., Hinckly, K.M., Jeffries, J.B., Hanson, R.K., "Pulse Detonation Engine Characterization and Control Using Tunable Diode-Laser Sensors," Journal of Propulsion and Power, Vol. 19, No. 4, July-August 2003.

[3] Shen, I., Adamson, T.C., "Theoretical Analysis of a Rotating Two Phase Detonation in a Rocket Motor,” The University of Michigan, March 1973.

[4] Bykovskii, F.A., Zhdan, S.A., Vedernikov, E.F., "Continuous Spin Detonations," Journal of Propulsion and Power, Vol. 22, No. 6, pp. 1204-1216, 2006.

[5] Wolański,P., "Detonative Propulsion" Proceedings of the Combustion Institute, Vol. 34, 2013,125,158. DOI: 10.1016/j.proci.2012.10.005.

[6] Heister, HY.D. and Stechmann, D.P., "Survey of Rotating Detonation Wave Combustor Technology and Potential Rocket Vehicle Applications," AIAA 2014-3902. DOI: $10.2514 / 6.2014-3902$.

[7] Kailasanth, K., "Recent Developments in the Research on Rotating-Detonation-Wave Engines," AIAA 2017-0784. DOI: 10.2514/6.2017-0784.

[8] Rankin, B. A., Fotia, M.L., Naples, A, G., Stevens, C. A., Hoke, J. L., Kaemming, T. A., Theuerkauf, S. W., and Schauer, F. R., "Overview of Performance, Application, and Analysis of Rotating Detonation Engine Technologies," Journal of Propulsion and Power, Vol. 33, No. 1, 2017,pp.131-143. DOI: 10.2514/1.B36303.

[9] Kailasanath, K., and Schwer,D.A., "High Fidelity Simulations of Pressure-Gain Combustion Devices Based on Detonations," Journal of Propulsion and Power, Vol.33, No.1, 2017, pp.153-162. DOI: 10.2514/1.B36169.

[10] Kurosaka, M., and Tsuboi, N., "Spinning detonation, cross-currents, and Chapman-Jouguet velocity," Journal of Fluid Mechanics, Vol. 756, 2014, pp. 728-757. DOI: 10.1017/jfm.2014.460.

[11] Schwer, D.A., Kailasanath, K., "Feedback into Mixture Plenums in Rotating Detonation Engines," AIAA 2012-0617. DOI:10.2514/6.2012-617.

[12] Shephard J.E., Kasahara, K., "Analytical Models for the Thrust of a Rotating Detonation Engine," GALCIT Report FM2017.001, September 2017. 
[13] Davidenko, D.M., Gokalp, I., Kudryavstev A.N., "Numerical Study of the Continuous Detonation Wave Rocket Engine," 15th International Space Planes and Hypersonic Systems and Technologies Conference. Dayton, OH. AIAA 2008-2680.

[14] Hishida, Manabu, Fujiwara, T., Wolanski, P., "Fundamentals of Rotating Detonations," Shock Waves, 19, 2009.

[15] Cao, H., "Analytical Parametric Analysis of Continuous Rotating Detonation EjectorAugmented Rocket Engine,” MS Thesis, University of Texas at Arlington, 2011.

[16] Smith, R.D. and Stanley, S.B., "Experimental Investigation of Continuous Detonation Rocket Engines for In-Space Propulsion," AIAA 2016-4582.

[17] Shank, J.C., "Development and Testing of a Rotating Detonation Engine Run on Hydrogen and Air," MS Thesis, Air Force Institute of Technology, 2012.

[18] Hargus, W.A., Schumaker, S.A., Paulson, E.J., and Danczyk, S.A., “Air Force Research Laboratory Rotating Detonation Rocket Engine Development," AIAA 2018-4876.

[19] Bohon, M.D., Bluemnery, R., Paschereitz, C.O., and Gutmark, E.J., "Experimental Study of Reactants Mixing in Model Rotating Detonation Combustor Geometries," AIAA 20174739.

[20] Edwards, A.C., Sherman, W.D., and Breidenthal, R.E., "Turbulent Mixing in Tubes with Transverse Injection," AICHE Journal, Vol. 31, No. 3, 1985, pp. 516-518.

[21] Breidenthal, R.E., Tong, K.O., Wong, G.S., Hamerquist, R.D. and Landry, P.B.," Turbulent Mixing in Two-Dimensional Ducts with Transverse Jets" AIAA Journal, Vol.24, No. 11, 1986, pp.1867-1869. DOI: 10.2514/3.9537

[22] Breidenthal, R.E., Buonadonna, V.R., and Weisbach, M.F., "Molecular Mixing via Jets in Confined Volumes," J. Fluid Mech., Vol. 219, pp. 531-544, 1990.

[23] Koch, J., "Summary of 1-D Detonation Analysis," December 2017.

[24] Wilhite, J.M., "Investigation of Various Novel Air-Breathing Propulsion Systems,” MS Thesis, University of Cincinnati, November 2016.

[25] Kuo, K., "Principles of Combustion,” McGraw-Hill, Boston, 2000.

[26] Knystautas, R., Guirao, C., Lee, J.H., Sulmistras, A., "Dynamics of Shock Waves, Explosions, and Detonations: Measurements of Cell Size in Hydrocarbon-Air Mixtures and Predictions of Critical Tube Diameter, Critical Initiation Energy, and Detonability Limits," AIAA, 1984. 
[27] Vutthivithayarak, R., Braun, E.M., Lu, F.K., "Examination of the Various Cycles for Pulse Detonation Engines," 47th AIAA/ASME/SAE/ASEE Joint Propulsion Conference \& Exhibit, AIAA Paper 2011-6064, July-Aug. 2011.

[28] Zabetakis, M.G., "Flammability Characteristics of Combustible Gases and Vapors," Bulletin 627, U.S. Bureau of Mines, 1965.

[29] Wolanski, P., Kauffman, C.W., Sichel, M., and Nicholls, J.A., "Detonation of methane-air mixtures," Symposium (International) on Combustion. 18. 1651-1660, 1981. DOI: 10.1016/S0082-0784(81)80169-5.

[30] Boening, J. A., Heath, J. D., Byrd, T.J., Koch, J. V., Mattick, A. T., Breidenthal. R. E., Knowlen, C., and Kurosaka, M., "Design and Experiments of a Continuous Rotating Detonation Engine: Wave Generator and Modulated Fuel-Oxidizer Mixing," AIAA 20164966.

[31] Stevens, C.A., Fotia, M.L., Hoke, J.L., Schauer, F.R., "Comparison of Transient Response of Pressure Meaurement Techniques with Application to Detonation Waves," AIAA 20151102, January 2015.

[32] Frolov, S.M., Basevich, V.Y., Aksenov, V.S., Polikhov, S.A., "Detonation Initiation by Controlled triggering of Electric Discharges," Journal of Propulsion and Power, Vol. 19, No. 4, July-August 2003.

[33] Heiser, W., and Pratt, D., "Thermodynamic Cycle Analysis of Pulse Detonation Engines," Journal of Propulsion and Power, Vol. 18, No. 1, January-February 2002.

[34] Schauer, F.R., Miser, C.L., Miser, K.C., Bradley, R.P., Hoke, J.L., “Detonation Initiation of Hydrocarbon-Air Mixtures in a Pulse Detonation Engine," AIAA 2005-1343, January 2005.

[35] Lu, J., Zheng, L., Wang, Z., Peng, C., \& Chen, X., “Thrust measurement method verification and analytical studies on a liquid-fueled pulse detonation engine," Chinese Journal of Aeronautics, 27(3), 497-504, 2014.

[36] Schwer, D.A., Kailasanath, K., "Feedback into Mixture Plenums in Rotating Detonation Engines," 50th Aerospace Sciences Meeting. Nashville, TN, AIAA 2012-0617, 2012.

[37] Voitsekhovskii, B.V., Mitrofanov, V.V., Topchian, M.E., "Investigation of the Structure of Detonation Waves in Gases," Symposium (International) on Combustion, 12(1), 829-837, 1969.

[38] Edwards, B. D., "Maintained detonation waves in an annular channel: A hypothesis which provides the link between classical acoustic combustion instability and detonation waves," Symposium (International) on Combustion, Vol. 16, pp. 1611-1618, 1977. 
[39] Suchocki, J.A., "Operational Space and Characterization of a Rotating Detonation Engine Using Hydrogen and Air,” MS Thesis, The Ohio State University, 2012.

[40] Russo, R.M., King P.I., Schauer, F.R., Thomas, L.M., "Characterization of Pressure Rise Across a Continuous Detonation Engine," Joint Propulsion Conference, AIAA-2011-6046, June 2011.

[41] Boening, J.A., "Initiation of Orderly Spinning Detonation Waves via Phased Sparking," MS Thesis, University of Washington, 2016.

[42] Kiyanda, C.B., Connolly-Boutin, S., Joseph, V., Mi, X., Ng, H.D., Higgins, A.J., "Small Sized Rotating Detonation Engine: Scaling and Minimum Mass Flow Rate," 26th ICDERS, July-August 2017.

[43] Yao, S., Zhang, S., Wang, J., "Effects of Pre-ignition Conditions on Continuous Detonation Engine," 26th ICDERS, July-August 2017.

[44] Wheeler, E.A., "Experimental Investigation of Throatless Supersonic Exhaust Velocity in a Rotating Detonation Engine," MS Thesis, University of Washington, 2017.

[45] Hansmetzger, S., Zitoun, R., Vidal, P., "Detonation Regimes in a Small-Scale RDE," 26th ICDERS, July-August 2017.

[46] Knowlen, C., Kurosaka, M., "Orderly Wave Initiation in a Rotating Detonation Engine," 26th ICDERS, July-August 2017.

[47] Boening, J. A., Heath, J. D., Byrd, T.J., Koch, J. V., Mattick, A. T., Breidenthal. R. E., Knowlen, C., and Kurosaka, M., "Design and Experiments of a Continuous Rotating Detonation Engine: Wave Generator and Modulated Fuel-Oxidizer Mixing," AIAA 20164966.

[48] Heath, J.D., "Generating Detonation Waves in an Annulus via Phased Adiabatic Shocks," M.S.A.A. Thesis, W.E.B. Dept. of Aeronautics \& Astronautics, University of Washington, 2015.

[49] Wheeler, E.A., Knowlen, C., and Kuroisaka, M., "Supersonic Exhaust Velocity in a Rotating Detonation Engine with Constant Area Annulus," AIAA 2018-0884. DOI: 10.2514/6.2018-0884.

[50] Koch, J.V., Washington, M.R., Kurosaka, M., and Knowlen, C., "Operating Characteristics of a CH4/O2 Rotating Detonation Engine in a Backpressure Controlled Facility," AIAA 2018-Xxxx.

[51] Edwards, A.C., Sherman, W.D., Breidenthal, R.E., "Turbulent Mixing in Tubes with Transverse Injection,” AIChE Journal, Vol. 31, No. 3, pp 516-517, March 1985. 
[52] Breidenthal, R.E., Tong, K., Wong, G.S., Hamerquist, R.D., Landry, P.B., "Turbulent Mixing in Two-Dimensional Ducts with Transverse Jets," AIAA Journal, Vol. 24, No. 11, pp 1867-1869, November 1986.

[53] Coquart, L., Sipp, D., Jacquin, L., "Mixing induced by Rayleigh-Taylor instability in a vortex," Physics of Fluids 17, 021703, January 2005.

[54] Bond, D., Johari, H., "Impact of buoyancy on vortex ring development in the near field," Exp Fluids 48, pp. 737-745, 2010.

[55] Alahyari, A., Longmire, E.K., "Dynamics of Experimentally Simulated Microbursts," AIAA Vol., 33, No. 11, November 1995.

[56] Sohn, S., "Late-Time Vortex Dynamics of Rayleigh-Taylor Instability," Journal of the Physical Society of Japan 80, 084401, August 2011.

[57] Donnadieu, C., Ortiz, S., Chomaz, J., Billant, P., "Three-dimensional instabilities and transient growth of a counter-rotating vortex pair," Physics of Fluids 21, 094102, September 2009.

[58] Read, K.I., "Experimental Investigation of Turbulent Mixing by Rayleight-Taylor Instability," Physica 12D, pp. 24-58, 1984.

[59] Dixit, H.N., Goveindarajan, R., "Vortex-induced instabilities and accelerated collapse due to inertial effects of density stratification," J. Fluid Mech., Vol. 646, pp. 415-439, February 2010.

[60] Sharp, D.H., “An Overview of Rayleigh-Taylor Instability,” Physica 12D, pp. 3-18, 1984. 
This page was intentionally left blank 


\section{APPENDIX A}

\section{Pre-Run Checklist: $\quad$ Date:___ Run \#:___ Initials:}

\section{Physical Checks:}

- Shop air turned "on", valve above the air hose should be in upwards facing position

- Gas cylinders connected: N2 6-pack, O2, $\mathrm{H} 2(\mathrm{CH} 4)$

- Before KT-300 VACUUM PUMP OPERATION

○ Check oil level: $3 / 8$ " above bottom of sight glass

- Close final dump tank water drain valves (bottom) and both vent valves (top)

- With pump isolation valve open, turn on pump (turn off if motor struggles), check oil again

- If open, close water drain valves in exhaust lines

- Record Butterfly Valve setting:

- Were there any instrumentation problems in the last run? Replace if necessary.

\section{Pre-Run VI Actions:}

- Close all unnecessary programs besides the LabView VI

- Validate run control sequence with valve control box set to: DIG DISABLE

- Validate all sensors are functional (if not fixable, note in instrument log - "Line Names File.xls"

- Validate ram lab CRDE VI is running and armed (via RDP) and seeing correct pressure transducers

- Check that data files from prior run are removed from "Data Files" folder

- Make new run folder (XXX) and backup folder (20xx-xx-xx-run\#)

- Update the run comments file with any instrumentation changes or notes

- Open CRDE Lab VI: CRDE_19

○ Open RAM Lab VI: CRDE_RAM_DAQ_2

\section{Pre-Run Physical Actions:}

- Confirm nominal operation of $\mathrm{H} 2 / \mathrm{O} 2$ flow control in prior run; if not,

- Readjust regulators if flow ratio is off

- Cycle $\mathrm{H} 2 *$ and $\mathrm{O} 2$ valves (switch 15 \& 16) if valve hang up detected

- Make sure the $\mathrm{H} 2$ line is vented upstream before cycling valve \#15!

- Power cycle the signal transfer box (if the DAS is not reading correct values)

- Set up camera(s) (if being used)

- Check vacuum is complete ( $<5 \mathrm{kPa}$ in reactor)

- Turn water on to PCB's

\section{Final Actions Before the run:}

- Verify wave number is selected to the correct value $(\mathrm{N}=1,2,3$, or 4$), \mathrm{N}=$

- Verify wave controller power supply is on at 1657 volts

- Shut off the KT-300 VACUUM PUMP (CLOSE VALVE BEFORE SHUTTING OFF PUMP)

- Start camera(s) (if being used)

- Validate spark frequency function generator is at desired frequency: $\mathrm{Hz}$ 
Pre \& Post Run Data to Record
Record:
$\underline{\text { Initial }}$
Final
Cylinders Used
- N2 6-pack cylinder(s) pressure:
$\mathrm{O} 2$ cylinders pressure:
p psig
kPa

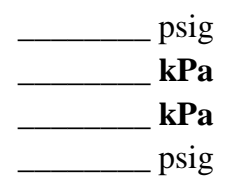
psig
$\mathrm{H} 2$ cylinders pressure:
$\mathrm{kPa}$
kPa
N2 (B) cylinders(s) pressure:
CHECK if outer wall temperature is below $80^{\circ} \mathrm{C}$
psig
psig

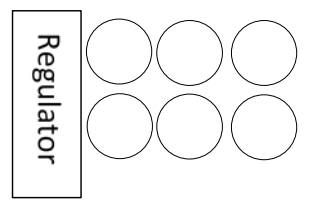
Close both doors to the shockwave reactor lab (210/239)
Set switch on solenoid controller box to: DIG ENABLE
Safety Glasses!

NOTE: in case of no-ignition, manually fill system to 70-100 $\mathrm{kPa}$ with $\mathrm{N2}$ by opening switch 4 and monitoring VI gauges

\section{Post-Run Checklist:}

Immediately after the run:

- Turn off digital disable switch on Solenoid Controller box after all automatic valve actions have stopped ( 20 $\underline{\mathrm{sec})}$

Over the next several minutes:

\section{If no detonation}

○ Purge system by back filling with $\mathrm{N} 2$ up to at least $50 \mathrm{kPa}$ (use valve 4 if minimum system pressure not reached in experiment)

○ Open room vent ball valve above the KT-300 pump SLOWLY to allow the SWR to fill with air from the room

\section{If detonation}

○ Open room vent ball valve above the KT-300 pump SLOWLY to allow the SWR to fill with air from the room

\section{In all cases}

○ Use KT-300 pump to evacuate purge gas to: $<26$ in- $\mathrm{Hg}$ or $10 \mathrm{kPa}$

- After purge evacuation, open Drain Valves at Front and Rear of Dump Tank

- Note final pressure in N2 6-pk manifold

$\circ \quad$ Close all N2 cylinders on 6-pk and its manifold isolation valve

○ Open water drain valves on KT-300 exhaust lines

- Shut off PCG water

Check that pressure in the dump tank is atmospheric BEFORE continuing!

○ Vent Hydrogen Manifold to the roof after closing $\mathbf{H} 2$ cylinders

- Vent Oxygen Manifold to the room

- Place the data files into the correct sub-folder after the run has completed $(<1 \mathrm{~min})$

- Run MatLab program to process data

On as needed basis

- Drain water from KT-300 crankcase (may be hot!)

- Separate water from vacuum oil using oil circulation pump (pump 5 minutes, drain — repeat if necessary)

\section{Gas Check List for Fuel and Oxidizer:}

Keep 1 bottle full and the other bottle empty. Equalize the pressure in both bottles. NEVER USE 2 FULL BOTTLES 


\section{APPENDIX B}

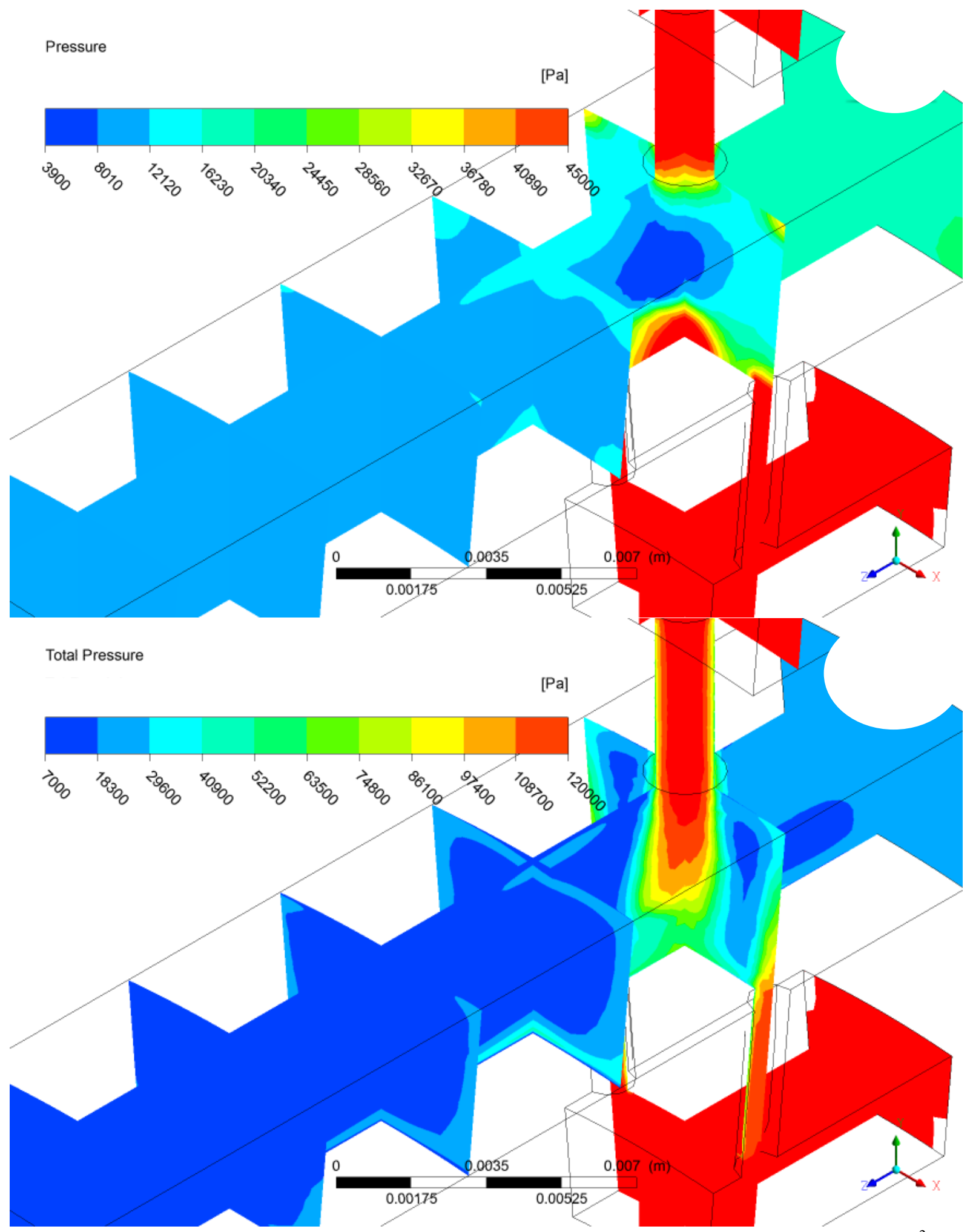

Figure B.1 Static (top) and total (bottom) pressure contours for 76-mm-RDE at mass flux $30 \mathrm{~kg} / \mathrm{s} / \mathrm{m}^{2}$. 


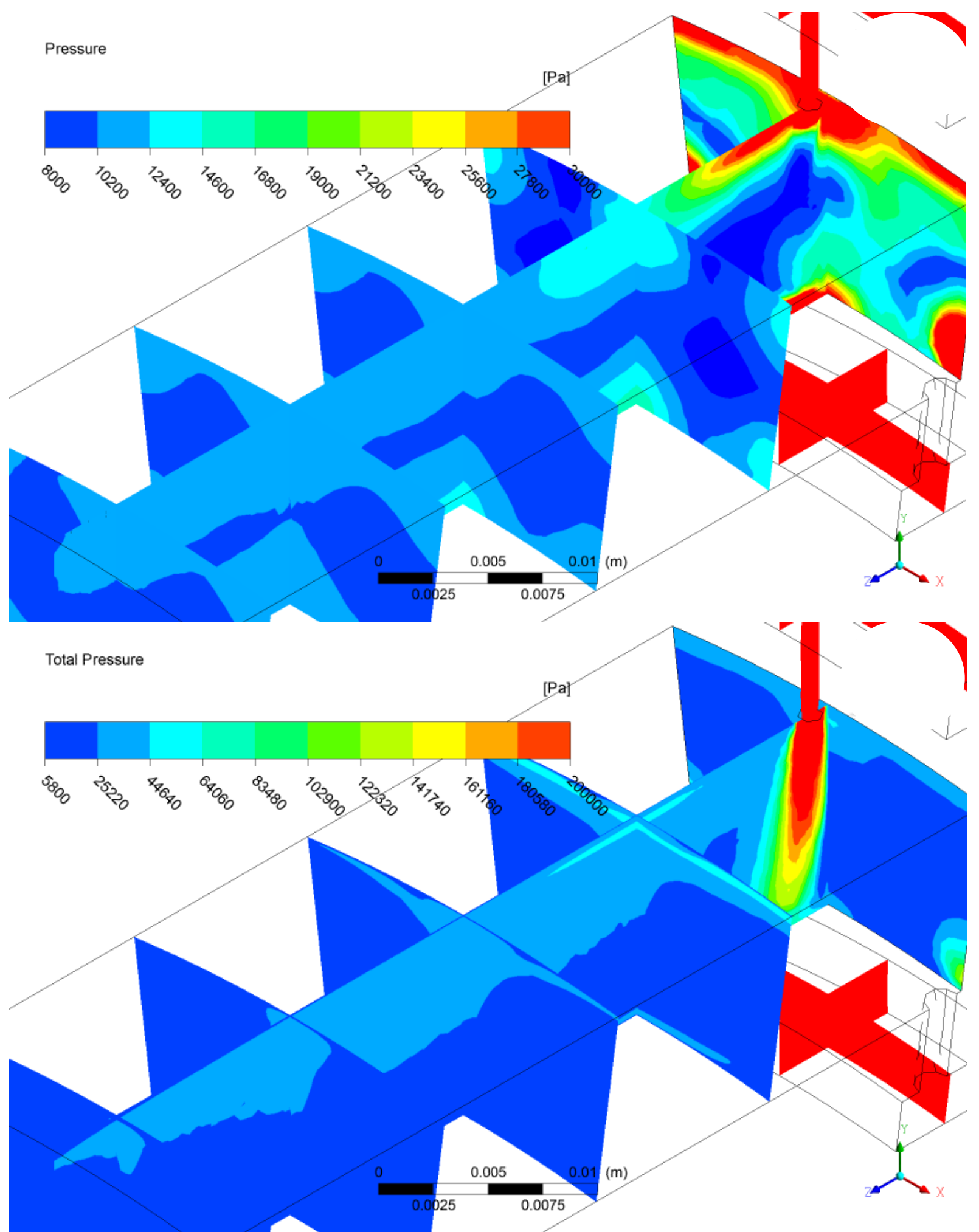

Figure B.2 Static (top) and total (bottom) pressure contours for 154-mm-RDE at mass flux $30 \mathrm{~kg} / \mathrm{s} / \mathrm{m}^{2}$. 
Table B.1 Average pressure summary for 76-mm-RDE at mass flux $30 \mathrm{~kg} / \mathrm{s} / \mathrm{m}^{2}$ (Injection scheme 2-1-3).

\begin{tabular}{c|cccc}
\hline \multirow{2}{*}{$\mathrm{z} / \mathrm{h}$} & Static Pressure & Total Pressure & \multirow{2}{*}{ Pressure Ratio } \\
\cline { 2 - 4 } & 122 & 122 & 1.00 \\
-1.27 & 88 & 89 & 1.00 \\
0 & 86 & 102 & 1.19 \\
1 & 12 & 19 & 1.63 \\
2 & 11 & 16 & 1.47 \\
3 & 11 & 15 & 1.39 \\
4 & 10 & 14 & 1.38 \\
& & & \\
0 (fuel) & 51 & 73 & 1.44 \\
0 (oxidizer) & 39 & 84 & 2.12 \\
\hline
\end{tabular}

Table B.2 Average pressure summary for 154-mm-RDE at mass flux $30 \mathrm{~kg} / \mathrm{s} / \mathrm{m}^{2}$ (Injection scheme 1-1-3).

\begin{tabular}{|c|c|c|c|}
\hline \multirow{2}{*}{$\mathrm{z} / \mathrm{h}$} & Static Pressure & Total Pressure & \multirow{2}{*}{ Pressure Ratio } \\
\hline & \multicolumn{2}{|c|}{$\mathrm{kPa}$} & \\
\hline-0.1 & 962 & 963 & 1.00 \\
\hline 0 & 601 & 671 & 1.12 \\
\hline 1 & 11 & 23 & 2.12 \\
\hline 2 & 11 & 18 & 1.71 \\
\hline 3 & 11 & 16 & 1.54 \\
\hline 4 & 10 & 15 & 1.49 \\
\hline 0 (fuel) & 480 & 635 & 1.32 \\
\hline 0 (oxidizer) & 445 & 779 & 1.75 \\
\hline
\end{tabular}



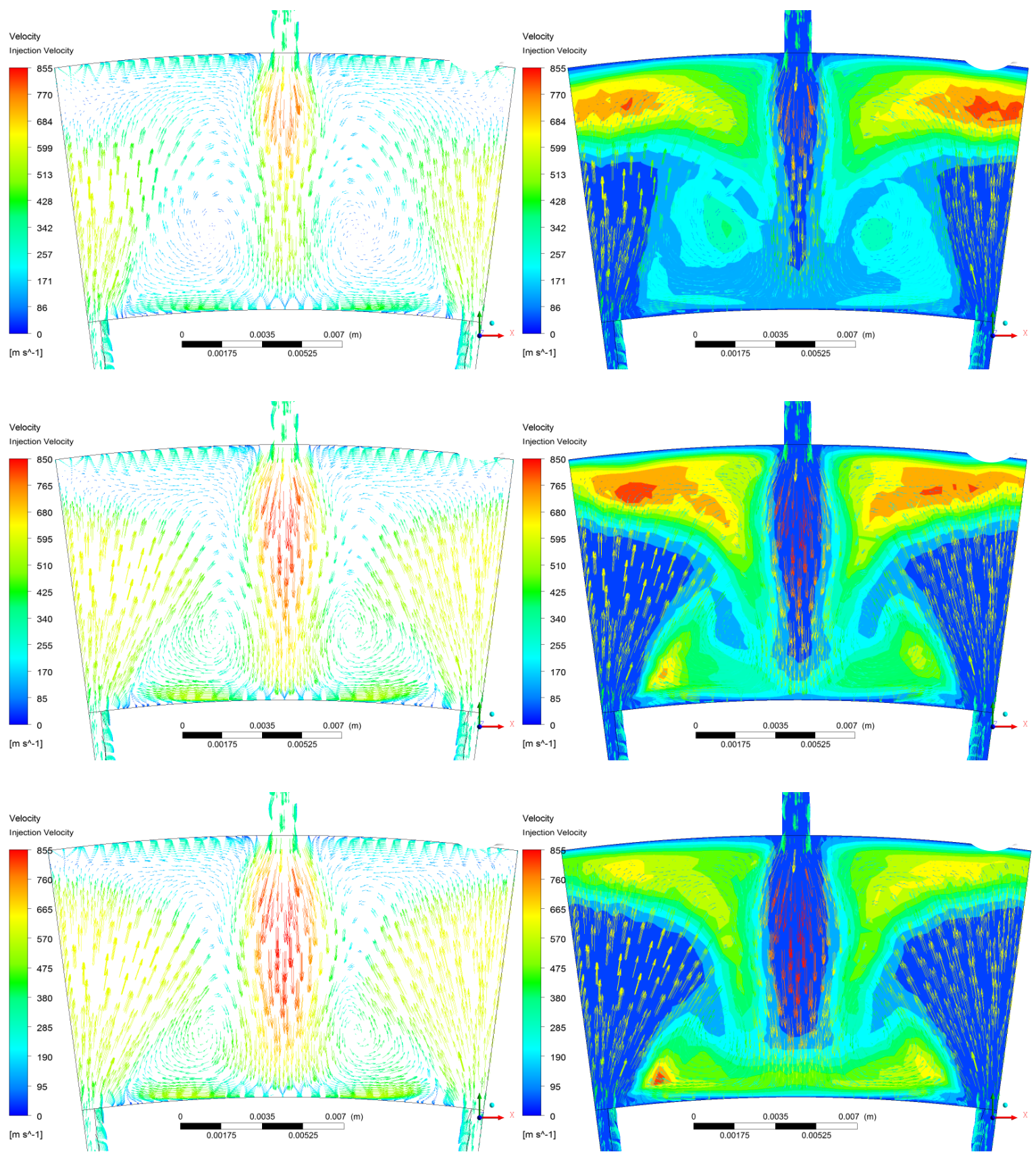

Figure B.3 Velocity profiles (left) and overlaid TKE contours (right) of different mass flux $10 \mathrm{~kg} / \mathrm{s} / \mathrm{m}^{2}$ (top) $20 \mathrm{~kg} / \mathrm{s} / \mathrm{m}^{2}$ (middle), and $20 \mathrm{~kg} / \mathrm{s} / \mathrm{m}^{2}$ (bottom) cases for 154-mm-RDE. Injection scheme 1-1-1 (top), 1-1-2 (middle), and 1-1-3 (bottom). 


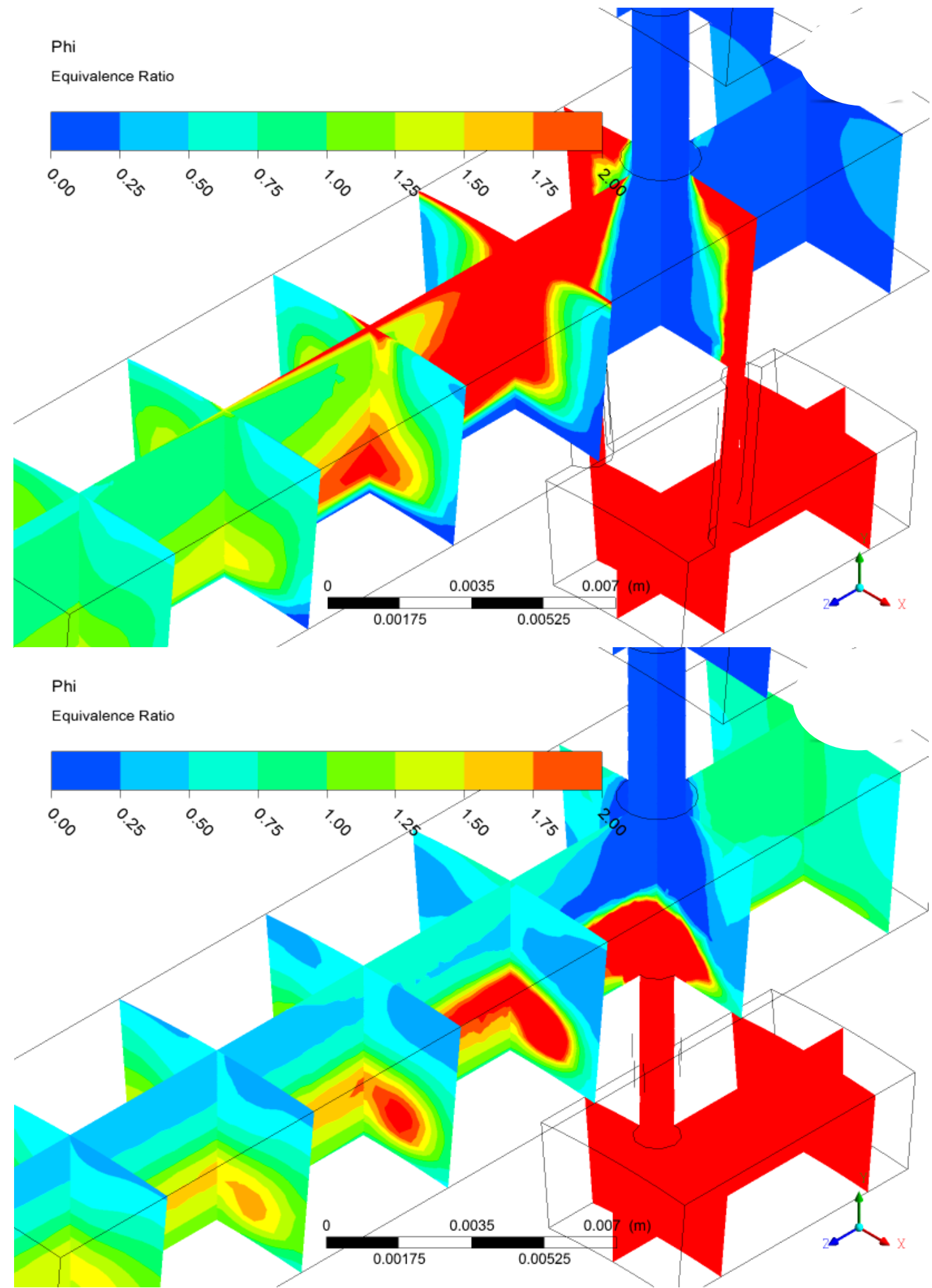

Figure B.4 Equivalence ratio contours for 76-mm-RDE. Staggered $30 \mathrm{~kg} / \mathrm{s} / \mathrm{m}^{2}$ (top) and impinged 270 $\mathrm{kg} / \mathrm{s} / \mathrm{m}^{2}$ (bottom). Injection scheme 2-1-3 (top) and 2-2-4 (bottom). 
This page was intentionally left blank 


\section{VITA}

Malik Washington obtained his Bachelor of Science in Aeronautical Engineering at the United States Air Force Academy in May of 2017 before attending the University of Washington in the Autumn of 2017. 